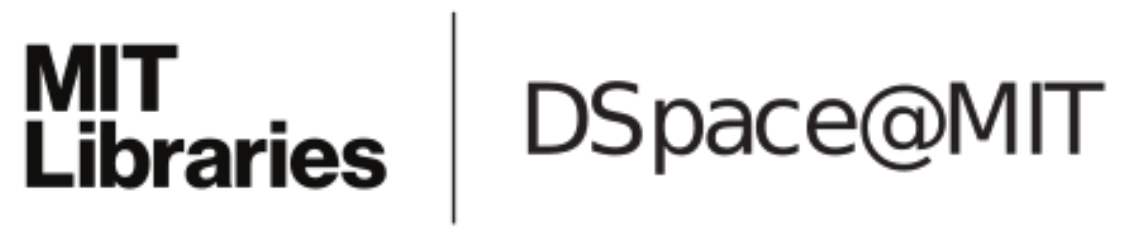

\author{
MIT Open Access Articles
}

Knockout of the gene encoding the extracellular matrix protein SNED1 results in early neonatal lethality and craniofacial malformations

The MIT Faculty has made this article openly available. Please share how this access benefits you. Your story matters.

Citation: Barqué, Anna et al. "Knockout of the gene encoding the extracellular matrix protein SNED1 results in early neonatal lethality and craniofacial malformations." Developmental Dynamics 250, 2 (October 2020): 274-294. (C) 2020 American Association of Anatomists

As Published: http://dx.doi.org/10.1002/dvdy.258

Publisher: Wiley

Persistent URL: https://hdl.handle.net/1721.1/131167

Version: Author's final manuscript: final author's manuscript post peer review, without publisher's formatting or copy editing

Terms of use: Creative Commons Attribution-Noncommercial-Share Alike 


\title{
Knockout of the gene encoding the extracellular matrix protein SNED1 results in early neonatal lethality and craniofacial malformations
}

\author{
Anna Barqué ${ }^{1}$, Kyleen Jan ${ }^{1}$, Emanuel De La Fuente ${ }^{2}$, Christina L. Nicholas ${ }^{2,3}$, Richard O. Hynes ${ }^{4,5^{*}}$, \\ Alexandra $\mathrm{Naba}^{1, *}$
}

1: Department of Physiology and Biophysics, College of Medicine, University of Illinois at Chicago, Chicago, Illinois, USA

2: Department of Orthodontics, College of Dentistry, University of Illinois at Chicago, Chicago, Illinois, USA

3: Department of Anthropology, College of Liberal Arts and Sciences, University of Illinois at Chicago, Chicago, Illinois, USA

4: Koch Institute for Integrative Cancer Research and Department of Biology, Massachusetts Institute of Technology, Cambridge, Massachusetts, USA

5: Howard Hughes Medical Institute, Massachusetts Institute of Technology, Cambridge, Massachusetts, USA

\section{* Correspondence:}

Alexandra Naba

Department of Physiology and Biophysics, University of Illinois at Chicago, Chicago, IL, USA

Email: anaba@uic.edu

Phone: 312-355-5417

Richard O. Hynes

Koch Institute for Integrative Cancer Research and Department of Biology, Massachusetts Institute of Technology, Cambridge, MA, USA

Email: rohynes@mit.edu

Phone: 617-253-6422

Running title: Snedl knockout causes craniofacial anomalies

Keywords: Knockout mouse, neural crest cells, craniofacial features, mandible, geometric morphometrics

\section{Funding information}

This work was funded in part by the Howard Hughes Medical Institute, of which ROH is an Investigator, a DOD Innovator Award W81XWH-14-1-0240 (ROH), the NIH/NCI Tumor Microenvironment Network U54-CA163109 (ROH), in part by the core grant to the Koch Institute (NIH/NCI P30CA14051) and by a start-up fund from the Department of Physiology and Biophysics at the University of Illinois at Chicago to AN. AB is the recipient of a Provost Graduate Research Award and an Award for Graduate Research from the UIC Graduate College, KJ is the recipient of a UIC Honors College Research Grant and an award from the LAS Undergraduate Research Initiative at UIC, EDLF is the recipient of a UIC L@S GANAS research fellowship. 


\section{Abstract}

Background: The extracellular matrix (ECM) is a fundamental component of multicellular organisms that orchestrates developmental processes and controls cell and tissue organization. We previously identified the novel ECM protein SNED1 as a promoter of breast cancer metastasis and showed that its level of expression negatively correlated with breast cancer patient survival. Here we sought to identify the roles of SNED1 during murine development.

Results: We generated two novel Sned 1 knockout mouse strains and showed that Snedl is essential since homozygous ablation of the gene led to early neonatal lethality. Phenotypic analysis of the surviving knockout mice revealed a role for SNED1 in the development of craniofacial and skeletal structures since Sned 1 knockout resulted in growth defects, nasal cavity occlusion, and craniofacial malformations. Sned 1 is widely expressed in embryos, notably by cell population undergoing epithelial-to-mesenchymal transition such as the neural crest cells. We further show here that mice with a neural-crest-cell-specific deletion of Sned 1 survive, but display facial anomalies partly phenocopying the global knockout mice.

Conclusions: Our results demonstrate requisite roles for SNED1 during development and neonatal survival. Importantly, the deletion of 2q37.3 in humans, a region that includes the SNED1 locus, has been associated with facial dysmorphism and short stature. 


\section{Introduction}

The extracellular matrix (ECM) is a fundamental component of multicellular organisms. It is a complex and dynamic assembly of hundreds of proteins that provides support to cells and instructs their behaviors. ${ }^{1-3}$ Mutations in ECM genes or changes in ECM composition, architecture or abundance have been shown to cause or accompany a plethora of diseases including fibrosis, renal, skeletal and vascular diseases, and cancers. ${ }^{3-6}$ Knockout mouse models have allowed the identification of the instrumental roles of ECM proteins and ECM-protein receptors at multiple steps of embryonic development. ${ }^{7-15}$ Knockout of several sub-units of the ECM receptors of the integrin family causes early embryonic lethality and diverse developmental defects. ${ }^{16,17}$ More specifically, and of relevance to the present study, ECM proteins including collagen, fibronectin, tenascin-C, versican, and aggrecan have been shown to regulate important features such as the migration of neural crest cells (NCCs) that will contribute to form craniofacial structures. ${ }^{18-21}$ In addition, other ECM components such as tenascin-C, biglycan, and fibromodulin have been reported to be involved in jaw development and the formation of the temporomandibular joint. ${ }^{22-24}$

The murine gene encoding the ECM protein SNED1 was cloned by Leimeister and collaborators in 2004 from renal stromal cells..$^{25}$ In the original publication, the authors used in-situ hybridization and showed that Sned1 is broadly expressed throughout development, mainly in the ectoderm, neural-crest, and mesodermal derivatives including kidneys, adrenal glands, lungs, skeleton, limbs, and the head region. ${ }^{25}$ Yet, the functions of SNED1 are, until now, unknown. In a proteomic screen comparing the ECM composition of poorly and highly metastatic human mammary tumors, we identified SNED1 as being more abundant in more aggressive mammary tumors. ${ }^{5}$ We further showed that SNED1 functioned as a metastatic promoter, since knocking down SNEDI's expression in tumor cells prevented metastasis. ${ }^{5}$ Importantly, we also demonstrated that $S N E D 1$ expression level was predictive of survival of hormonereceptor negative breast cancer patients. ${ }^{5}$ Additionally, Sned 1 was found to be up-regulated in murine pancreatic ductal adenocarcinoma (PDAC) cells cultured in a 3-dimensional ECM and which are more resistant to chemotherapeutic drugs, as compared with cells grown on a 2-dimensional substrate. ${ }^{26}$ Sned 1 knockdown was also shown to inhibit mutant-p53-driven murine PDAC cell invasion in vitro. ${ }^{27}$ While SNED1 has been shown to regulate cancer cell phenotypes, the roles of this protein in embryonic development and physiology remain to be identified. To address this question, we generated knockouts of Sned 1 in mice. Using these novel mouse models, we demonstrate here that Sned 1 is an essential gene, 
since Sned 1 knockout causes $\sim 61 \%$ early neonatal lethality between $\mathrm{p} 0$ and $\mathrm{p} 2$. Over the course of our study, some homozygous knockout mice survived. Interestingly, they were easily distinguishable from wild-type and heterozygous littermates because of their smaller size and craniofacial malformations. Considering the craniofacial phenotype observed in knockout mice and the pattern of expression of Sned1, we further sought to examine the potential role of SNED1 in the NCC population, which contributes to form most of the bones and cartilage of the head. ${ }^{28-31}$ To do so, we genetically ablated Sned 1 in the NCCs and observed that, while Sned $1^{N C C-/ N C C-}$ mice survived, they presented growth defects and craniofacial anomalies resembling malformations observed in the global knockout animals. Altogether, our results point to a role for SNED1 in multiple aspects of embryonic development, including the formation of craniofacial structures.

\section{Results}

\section{Snedl is conserved among all vertebrates and presents key features of ECM proteins}

The SNED1 ( Sushi, Nidogen and EGF-like Domains 1 ) gene, initially named SNEP (ㅁtromal Nidogen Extracellular matrix Protein), encodes a secreted protein composed of characteristic domains commonly found in ECM proteins ${ }^{32,33}$ including an amino-terminal NIDO domain, 15 EGF-like and calciumbinding EGF-like domains, one or two Follistatin-N-terminal-like (FOLN) domains (depending on the prediction algorithms ${ }^{34,35}$ ), a Complement Control Protein (CCP, also known as Sushi domain), and three Type III fibronectin (FN3) domains in the carboxy-terminal region of the protein (Figure 1). Interrogation of gene and protein databases revealed that orthologues of SNED1 are found in all sequenced vertebrates, including fish, reptiles, amphibians, birds, and mammals (Figure 1), but not in lower organisms. The evolution of vertebrates and mammals has been accompanied by the expansion of families of ECM proteins existing in lower organisms (such as the collagens) and by the appearance of novel ECM proteins, ${ }^{36-38}$ such as SNED1. This increasing complexity of the ECM has been concomitant with the appearance of novel structures including the neural crest and endothelium-lined vasculature in vertebrates or of mammary glands in mammals. ${ }^{39,40}$

In addition, sequence analysis has revealed the presence of an RGD motif in the carboxy-terminal region of the zebrafish sequence and two consensus potential binding sites, RGD and LDV, for integrins, ${ }^{41,42}$ which are known ECM protein receptors, in the amino-terminal region of mammalian sequences of SNED1 (Figure 1), suggesting that the protein has further evolved, likely to support the development of mammalian-specific structures, as has occurred for other ECM proteins. 


\section{Snedl knockout results in early neonatal lethality}

Snedl was targeted using the "knockout-first allele" construct designed through an effort supported by the Wellcome Trust Sanger Institute and the Knockout Mouse Project ${ }^{43}$. In a first approach, Sned1 knockout was achieved using an IRES:LacZ-trapping cassette ${ }^{43}$ inserted in the second intron of Snedl (Figure 2A). Using RT-qPCR on RNA extracted from mouse embryonic fibroblasts generated from mice

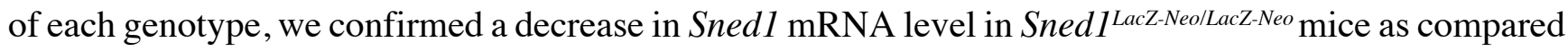
to Sned $1^{\text {WT/WT }}$ and Sned $1^{\text {WT/LacZ-Neo }}$ mice (Figure 2C).

We intercrossed heterozygous (Sned $1^{\text {WT/LacZ-Neo }}$ ) mice and examined 55 litters born from 16 different breeding pairs, representing a total of 300 pups, of which 202 were still alive at weaning. We observed that a significant number of pups died within 48 hours after birth, and, when carcasses could be retrieved for genotyping, we found that the percentage viability of knockout (Sned1 ${ }_{\text {LacZ-Neo/LacZ-Neo }}^{\text {) neonates was }}$ notably lower (38.3\%) in contrast to wild-type (73.0\%) and heterozygous (72.6\%) pups (Table 1). Of note, it is probable that we were not able to retrieve all carcasses, some having likely been cannibalized

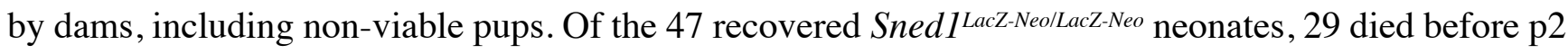
and only 18 were alive at weaning (p21 +/- 1 day), a sub-Mendelian ratio (8.9\% instead of the expected 25\%; $\chi^{2}=20.303$; two-tailed $\left.\mathrm{p}<0.0001\right)$ (Table 1). However, Mendelian ratios were observed for E18.5 embryos obtained by $\mathrm{C}$-section ( 6 litters analyzed, $\chi^{2}=1$; two-tailed $\mathrm{p}=0.607$ ), suggesting that lethality of knockout pups occurred either around or shortly after birth (Table 1).

\begin{tabular}{|l|c|c|c|c|}
\hline $\begin{array}{l}\text { Sned1 }^{\text {WT/LacZ-Neo }} \mathbf{x} \\
\text { Sned1 }^{\text {WT/LacZ-Neo }}\end{array}$ & Sned1 $^{\text {WT/WT }}$ & Sned1 $^{\text {WT/LacZ-Neo }}$ & Sned1 $^{\text {LacZ-Neo/LacZ-Neo }}$ & $\begin{array}{c}\chi^{2} \\
\boldsymbol{p}_{\text {-value }}\end{array}$ \\
\hline Expected & $25 \%$ & $50 \%$ & $25 \%$ & \\
\hline E18.5 & $14(27.5 \%)$ & $22(43.1 \%)$ & $15(29.4 \%)$ & 0.607 \\
\hline Recovered neonates & $74(24.7 \%)$ & $179(59.6 \%)$ & $\mathbf{4 7}(\mathbf{1 5 . 7 \% )}$ & \\
\hline Alive at weaning (p21) & $54(26.7 \%)$ & $130(64.4 \%)$ & $\mathbf{1 8}(\mathbf{8 . 9 \% )}$ & $<\mathbf{0 . 0 0 0 1}$ \\
\hline Percentage viability & $73.0 \%$ & $72.6 \%$ & $\mathbf{3 8 . 3 \%}$ & \\
\hline
\end{tabular}

Table 1. Numbers of expected and observed E18.5 embryos and 21-day-old pups, total number of recovered neonates, and percentage viability of each genotype obtained by crossing heterozygous Sned ${ }^{\text {WT/LacZ-Neo }}$ mice.

We then generated a conditional knockout mouse strain by crossing heterozygous Sned $1^{\text {WT/LacZ-Neo }}$ mice with mice expressing the Flp recombinase under the control of the actin promoter. This resulted in the excision of the IRES:LacZ and Neo cassettes, and the generation of a floxed allele (Sned ${ }^{f l}$, Figures $2 \mathrm{~A}$ 
and 2B). Sned ${ }^{f l f l}$ mice were indistinguishable from wild-type mice indicating that the excision of the gene-trap cassette reverted the phenotype. This strain will be useful to determine the function of SNED1 in a cell- or time-specific manner (see below). We further crossed conditional knockout mice with mice expressing the Cre recombinase under the control of a CMV promoter, causing to the deletion of exons 3 and 4 of the Snedl gene. This introduces a frameshift mutation that includes 43 aberrant amino acids followed by a premature stop codon that triggers nonsense-mediated decay of the truncated transcripts ${ }^{43}$ and ultimately generates a null allele (Sned $1^{-}$, Figures $2 \mathrm{~A}$ and $2 \mathrm{~B}$ ). Heterozygous mice (Sned $1^{\text {WT/-) }}$ ) were further intercrossed to generate homozygous null mice (Sned1 $\left.1^{-/}\right)$. In-situ hybridization on sagittal sections of E18.5 mice confirmed decreased expression of Snedl mRNA in the Sned1 ${ }^{-/-}$embryos as compared to Sned $1^{W T / W T}$ and Sned $1^{W T /-}$ embryos (Figure 2D, showing the primordium costal cartilage of the developing ribs).

We evaluated 13 litters born from 7 different breeding pairs of heterozygous mice (representing a total of 88 pups alive upon inspection of the cages on the expected date of birth, of which 63 were still alive at weaning). Similarly to what we observed with the gene-trap allele, a significant number of pups died within 48 hours after birth. When carcasses were retrieved for genotyping, we found that the percentage viability of knockout (Sned1 $\left.1^{-/}\right)$neonates was considerably lower $(38.5 \%)$ when compared to wild-type $(60.9 \%)$ and heterozygous (84.6\%) pups (Table 2). We further observed that at weaning (p21 +/- 1 day), null mice $\left(\right.$ Sned $\left.^{-/}\right)$were present in sub-Mendelian ratio $\left(7.9 \%\right.$, instead of the expected $25 \% ; \chi^{2}=12.492$; two-tailed $\mathrm{p}=0.0019$ ), indicating that null pups had died before that (Table 2). 5 litters were harvested by C-section to examine a total of 44 E18.5 embryos. As observed for the Sned $1^{\text {LacZ-Neo }}$ strain, Mendelian ratios were observed $\left(\chi^{2}=0.364\right.$; two-tailed $\left.\mathrm{p}=0.834\right)$ indicating that lethality of Sned $1^{-/-}$pups occurred around or shortly after birth (Table 2).

\begin{tabular}{|c|c|c|c|c|}
\hline Sned $1^{W T /-} \times$ Sned $1^{W T /-}$ & SnedI ${ }^{W T / W T}$ & Sned $1^{W T /-}$ & Sned1 $^{-/-}$ & $\underset{p \text {-value }}{\chi^{2}}$ \\
\hline Expected & $25 \%$ & $50 \%$ & $25 \%$ & \\
\hline E18.5 & $12(27.3 \%)$ & $20(45.5 \%)$ & $12(27.3 \%)$ & 0.834 \\
\hline Recovered neonates & $23(26.1 \%)$ & $52(59.1 \%)$ & $13(14.8 \%)$ & \\
\hline Alive at weaning (p21) & $14(22.2 \%)$ & $44(69.8 \%)$ & $5^{*}(7.9 \%)$ & 0.0019 \\
\hline Percentage viability & $60.9 \%$ & $84.6 \%$ & $38.5 \%$ & \\
\hline
\end{tabular}

Table 2. Numbers of expected and observed E18.5 embryos and 21-day-old pups, total number of recovered neonates, and percentage viability of each genotype obtained by crossing heterozygous Sned $1^{W T /}$ mice $\left(^{*} 1\right.$ mouse died at p22 and one at p24). 
Altogether, our data demonstrate that Snedl knockout, achieved using two approaches, results in early neonatal lethality, with around $61 \%$ penetrance (Tables 1 and 2 ).

Remarkably, the knockout mice (either Sned1 $1^{\text {LacZ-Neo/LacZ-Neo }}$ or Sned1 $1^{-/}$) who survived were easily distinguishable from wild-type and heterozygous littermates because of their smaller size and the shape of their head and snout. We thus sought to characterize in detail their phenotype to gain insight into the physiological roles of SNED1. Of note, crossing of Sned1 $1^{\text {LacZ-Neo/LacZ-Neo }}$ survivors revealed that surviving Snedl knockout mice were fertile although they produced less numerous and smaller litters. However, pups obtained by breeding Sned $1^{\text {LacZ-Neo/LacZ-Neo }}$ survivors displayed exacerbated phenotypes (in particular, even shorter snouts, under-developed or lacking mandibles; not shown) and only some of their progeny survived after weaning.

\section{Snedl knockout results in growth defects}

Our first observation was that knockout survivors (either Sned1 $1_{\text {LacZ-Neo/LacZ-Neo }}$ or Sned1 $1^{-/}$) were visibly smaller than wild-type and heterozygous littermates (Figure 3A). At p14, knockout pups weighed significantly less than wild-type and heterozygous littermates (Figures 3B and 3D). This reduced body weight was readily detectable in p0.5 neonates (Figure 3C). Suspecting skeletal anomalies, we acquired micro-computed tomography $(\mu \mathrm{CT})$ scans of these mice when they reached adulthood and measured the

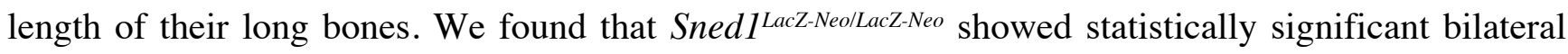
shortening of the humerus and femur compared to control and heterozygous littermates (Figures $3 \mathrm{E}$ and $3 \mathrm{~F}$ ) and of all other bones measured (ulna, radius, and tibia; data not shown). No significant differences were found between the wild-type and heterozygous groups.

\section{Sned1 knockout mice present craniofacial malformations}

Besides their small size, knockout survivors were also distinguishable from wild-type and heterozygous littermates by their shorter snouts and abnormal head shape. Thus, we examined the skulls of wild-type, heterozygous and Snedl knockout mice using $\mu \mathrm{CT}$ (Figure 4A). We measured the length of the different calvarial sutures including the nasal, frontal, and sagittal sutures (Figure 4A). Knockout mice showed statistically significantly shorter nasal sutures as compared to wild-type $(\mathrm{p}=0.004)$ or heterozygous $(\mathrm{p}=0.005)$ mice (Figure 4B, left panel). Frontal sutures did not differ significantly in length, although there was a trend towards shorter sutures in the knockout mice (Figure 4B, right panel). The length of 
the sagittal sutures, joining parietal bones of mesodermal origin, although tending to be shorter, did not show statistically significant differences between groups (data not shown).

We further employed geometric morphometric analysis, an approach that compares the relative positions of anatomical landmarks, ${ }^{44}$ to quantify complex 3-dimensional shape variations between mice of different genotypes. To do so, we positioned 22 traditional coordinate landmarks which were deemed to represent well overall cranial morphology and an additional 8 sliding semi-landmarks along the nasal suture, since this structure was particularly affected upon Snedl knockout (see Supplementary Method 1). Landmark configurations are then superimposed and compared using principal component analysis (PCA). PCA yielded 19 principal components, the first 4 of which represented over $5 \%$ of the overall variation. The first principal component (PC1) accounted for $30.05 \%$ of the overall variation and described differences in cranial vault shape and nasal bridge morphology. PC1 showed statistically significant differences across the three groups $(\mathrm{p}=0.027$, Figure 4C). A Tukey's honest significant difference (HSD) test revealed that the overall statistical significance in PC1 was driven by differences between the wild-type and knockout groups $(\mathrm{p}=0.048)$ and between the heterozygote and knockout groups $(\mathrm{p}=0.017)$ while the comparison of the heterozygous and wild-type mice was not statistically significantly different $(\mathrm{p}=0.99)$ (Figure 4D). Wireframes depicting the consensus cranium shape were drawn (Figure 4E, grey dots) and the average shapes of the extreme ends of the range of variation for PC1 were superimposed (Figure 4E, black dots). Animals at the minimum extreme of variation along PC1 (PC1 min, knockout mice) showed concavity of the nasal bridge, relatively shorter snouts, wider faces and neurocrania, and more supero-inferiorly expanded neurocrania (Figure 4E, upper panel). In contrast, those at the maximum extreme of variation along PC1 (PC1 max, wild-type and heterozygous mice) tended to show relatively flatter (supero-inferiorly) and medio-laterally narrower neurocrania, narrower midfaces (at the zygoma), and a more convex nasal bridge (Figure 4E, lower panel). Although the mechanisms by which SNED1 controls nasal bridge formation remain unknown, we can hypothesize that they contribute to the proper formation of the nasal septum, the cartilaginous structure that supports the ossified nasal bridge.

\section{Snedl knockout mice present asymmetric and occluded nasal cavities}


The observation that Snedl knockout mice present nasal bridge collapse prompted us to further evaluate the anatomy of the nasal cavity of the animals. In the cohort of adult mice, 2 out of the 5 knockout mice presented partial occlusion, defined as a contact between the soft tissues forming the wall of the airways, whereas none of the wild-type and heterozygous mice presented occlusion. In addition, 3 out of the 5 knockout mice presented markedly asymmetric nasal cavities, whereas 9 out of 10 heterozygous and all 4 wild-type mice had symmetrical cavities (Figure 4F, Supplementary Files 1-3). Interestingly, nasal occlusion is already present at birth, since the evaluation of a small cohort of neonates (p0.5) revealed that all knockout neonates analyzed presented at least partial nasal occlusion, in contrast to none of the wild-type animals, and only 3 out of the 15 heterozygotes studied (Figure 4G, Supplementary Files 46). Asymmetric nasal cavities and airway obstruction support the hypothesis that the Snedl knockout neonates may not survive because of impaired nasal respiration.

\section{Snedl knockout mice present under-developed mandibles}

Interestingly, we made the observation that some neonates ( $\mathrm{p} 0.5$ ) differed from others by the absence of milk in their stomach. Genotyping of the animals revealed that pups lacking a milk pocket were the

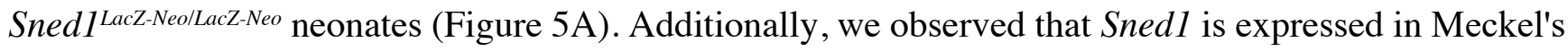
cartilage from which the mandibles develop (Figure 6E, see below), indicating a putative role of SNED1 during the formation of the lower jaw. Therefore, we further investigated variations in the mandibular structure of our mice using geometric morphometric analysis. PCA yielded 18 principal components, the first 7 of which represented greater than $5 \%$ of the overall variation. PC1 represented $25.49 \%$ of the variation and showed statistically significant differences across the three groups ( $\mathrm{p}=0.001)$ (Figure 5B). A Tukey's HSD test revealed that these differences were statistically significant between the wild-type and knockout groups $(\mathrm{p}=0.0002)$ and between the heterozygous and knockout groups $(\mathrm{p}<0.0001)$ while the comparison of the heterozygous and wild-type mice was not statistically significantly different $(\mathrm{p}=0.954)$ (Figure 5C). Mice at the maximum extreme of variation along PC1 (PC1 max, knockout mice) showed shorter mandibular condyles and smaller coronoid processes (Figure 5D, lower panel). In contrast, those at the minimum extreme of variation along PC1 (PC1 min, wild-type and heterozygous mice) who tend to display longer mandibular condyles and larger coronoid processes (Figure 5D, upper panel). Of note, we occasionally recovered dead Sned $1^{-/-}$neonates with extreme phenotype including complete lack of lower jaw (Figure 5E). Although at this point the mechanisms by which SNED1 controls mandible formation remain unknown, we hypothesize that, depending on severity, they may affect the ability of knockout mice to suckle or chew properly, as the mandibular condyle and coronoid 
process are attachment points for chewing muscles such as the masseter. The mandibular condyle is also an important growth site of the mandible, contributing to the elongation of the ramus. ${ }^{45}$ It will thus be interesting to determine whether the growth defects in Snedl knockout mice (Figure 3) are an indirect result of the decreased ability of knockout mice to eat or whether SNED1 plays a direct role during skeletal development.

\section{Sned1 is expressed in skeletal and craniofacial precursors}

We took advantage of the LacZ reporter gene expressed under the control of the Snedl promoter to determine in which tissues Snedl was expressed during development. Whole-mount LacZ staining revealed that the Snedl promoter is broadly active at the two timepoints examined, embryonic days 11.5 (Figure 6A) and 13.5 (Figure 6D). Many of the zones of expression conform with tissues that later show defects. At E11.5, LacZ staining was observed in the nasal process and the pharyngeal arches (Figure 6B), structures populated by cephalic neural crest cells (NCCs) that will contribute to form the skull and facial skeleton. ${ }^{46,47}$ At E11.5, LacZ staining was also detected in mesodermal derivatives including the ventro-medial cells of the somites (Figure 6C), which will undergo epithelial-to-mesenchymal transition (EMT) to form the sclerotomes which will, in turn, give rise to the vertebrae and the ribs. In addition, staining was detected in the lung bud (Figure 6C), as previously reported ${ }^{25}$ and in Rathke's pouch, a structure deriving from the oral ectoderm which will give rise to the adenohypophysis of the pituitary gland. At E13.5, staining was visible in the head region, in the derivatives of the pharyngeal arches, including the tongue and the Meckel's cartilage, which will contribute to the development of the mandible, the cartilage primordium of the nasal septum, and the vomeronasal organ, a chemoreceptive organ, part of the olfactory system (Figures 6E and 6G). Staining was also observed in the skeletal precursors of the humeral shaft and the scapula (Figure 6E), the vertebrae and the ribs (Figures 6D and 6G). We also observed that the LacZ staining intensity followed the antero-posterior axis, with the posterior somites being the last to differentiate and the most intensely stained (Figure 6G, right panel). Staining was also observed in the limb buds (Figures 6F and 6G), the choroid plexus (Figure 6G, middle and left panel), and in the mesenchyme surrounding the dorsal root ganglia. Our results confirm and extend the previously published study reporting the expression of Snedl using in-situ hybridization in the limb buds, tail, sclerotome, vertebrae and ribs, lung, kidney, adrenal gland, cerebellum, choroid plexus and head mesenchyme, ${ }^{25,48}$ and with gene expression data reporting Snedl expression in the mandible and frontonasal region of E10.5 to E12.5 embryos. ${ }^{49,50}$ 


\section{Deletion of Sned1 in the neural crest cells does not affect mouse survival}

The craniofacial skeleton has two embryonic origins; the cephalic NCCs form the anterior region of the skull, while the mesoderm contributes to the more posterior cranial bones. ${ }^{51,52}$ Interestingly, the length of the sagittal suture, formed by the parietal bones of mesodermal origin, was not statistically different between wild-type, heterozygous, and Snedl knockout mice (data not shown). Following induction, cephalic NCCs undergo EMT and migrate from the dorsal neural tube into the frontonasal region and pharyngeal arches, giving rise to most of the bone and cartilage of the head and neck. ${ }^{28-31}$ The mesenchyme of nasal, frontal, and sagittal sutures is also neural-crest derived. ${ }^{53}$ Because Snedl is expressed in structures that will form the craniofacial skeleton and NCC-derived tissues, including the nasal bone and the mandible, and because these structures are abnormal in Snedl knockout mice, we hypothesized that it may regulate NCC phenotype. Interestingly, the origin of vertebrates is concomitant with the appearance of the NCC population, ${ }^{39,54}$ and orthology analysis has found that orthologues of Snedl are found in all sequenced vertebrates, but not in lower organisms (Figure 1).

To assess the putative role of SNED1 in the NCCs, we ablated Snedl specifically from NCCs. To do so, we crossed mice carrying the conditional Snedl allele (Sned $l^{f l f f}$ ) with the Wntl-Cre2 transgenic mouse line (Figure 7A), ${ }^{55}$ an established model used for the study of neural crest development and its derivatives. We examined 32 litters born from 6 different breeding pairs (representing a total of 180 pups). Contrary to what we observed in the global Snedl knockout mice, the NCC-specific deletion of Sned 1 did not affect survival since mice were present in Mendelian ratios $\left(\chi^{2}=5.378\right.$; two-tailed $\left.\mathrm{p}=0.146\right)$ at weaning (p21 +/- 1 day, Table 3). These data suggest that the production of SNED1 by other cell types apart from the NCCs is essential for development.

\begin{tabular}{|c|c|c|c|c|c|}
\hline $\begin{array}{l}\text { Wnt1-Cre2; SnedI }{ }^{\text {WT/fl }} \mathbf{x} \\
\text { SnedI Illfl }^{\text {for }}\end{array}$ & Sned1 ${ }^{W T / f l}$ & SnedI ${ }^{f l f l}$ & $\begin{array}{c}\text { Wnt1-Cre2; } \\
\text { Sned1 }^{W T / f l} \\
\text { (Sned1 }^{W T / N C C-)}\end{array}$ & $\begin{array}{c}\text { Wnt1-Cre2; } \\
\text { Sned1 } \\
\text { (Snedlfl }^{\text {SCC-/NCC-) }}\end{array}$ & $\underset{p-v a l u e}{\chi^{2}}$ \\
\hline Expected & $25 \%$ & $25 \%$ & $25 \%$ & $25 \%$ & \\
\hline Alive at weaning (p21) & $57(31.7 \%)$ & $36(20.0 \%)$ & $46(25.5 \%)$ & $41(22.8 \%)$ & 0.146 \\
\hline
\end{tabular}

Table 3. Numbers of expected and observed 21-day-old pups of each genotype obtained by crossing Wnt1-Cre2; SnedI fllt with Sned $I^{f l l f l}$ mice. 


\section{Deletion of Sned 1 in the neural crest cells results in growth defects and craniofacial malformations partly phenocopying the effect of the global deletion of SnedI}

Since the global Snedl knockout resulted in growth defects, we also recorded possible differences in body weight and observed that one-month-old Sned $1^{N C C-N C C-}$ mice were smaller and weighed less than control and heterozygous littermates (Figure 7B). Importantly, and similarly to global knockout mice, Sned $1^{N C C-N C C-}$ mice could be easily distinguished because they presented craniofacial malformations, including shorter snouts and wider neurocranium (Figure 7C). To further examine variations in shape based on the genotype, we scanned the skulls of 30 adult mice using $\mu \mathrm{CT}$. We observed that the nasal bone was severely malformed upon deletion of Snedl in the NCCs (Figure 7D, arrow). Sned $1^{\text {NCC-NCCC- }}$ mice showed significantly shorter nasal sutures as compared to controls (Sned $1^{\text {WTfl }}$ and Sned $1^{f l f f}$ ) and heterozygotes (Sned1 ${ }^{\text {WTNCC-})}$ (Figure 7E). No statistically significant difference was observed in the length of the frontal suture between genotype groups, although there was a trend toward Sned $1^{\text {NCC-INCC- }}$ mice having shorter frontal sutures. Contrary to what we observed upon global Sned 1 knockout, sagittal sutures of Sned $1^{N C C-N C C C-}$ mice were also shorter as compared to control and heterozygous mice (data not shown).

We then applied the same standard geometric morphometric analysis as the one employed to characterize the craniofacial features of the global KO mice, to assess variations in the shape of the skulls of our cohort of control and NCC-specific knockout mice $(n=30)$. Principal component analysis yielded 29 PCs, the first 4 of which represented at least $5 \%$ of the total variation each. Analysis of the variance of the 4 different groups along the first 4 PC axes found statistically significant differences along PC2 ( $\mathrm{p}=0.003$; Figure 7F), which accounted for $11.75 \%$ of the overall variation. A Tukey's HSD test further revealed that Sned $1^{N C C-I N C C-}$ mice drove this variation, and was statistically significantly different from control (Sned $l^{\text {WTfll }} \mathrm{p}<0.0001$; Sned $\left.l^{f l f l} \mathrm{p}=0.001\right)$ and heterozygous mice $(\mathrm{p}<0.0001)$. As shown in Figure 7F, most of the Sned $I^{\text {NCC-INCC- }}$ mice cluster at the negative end of the range of variation along PC2 (PC2 min), which represents mice with more concave nasal bridges, relatively shorter snouts, and relatively wider crania (Figure 7H); the same morphological craniofacial features observed upon global Snedl deletion.

We also evaluated the anatomy of the nasal cavity in the NCC-specific Sned 1 knockout mice. Out of the 11 knockout mice, 3 presented partial occlusion of the nasal cavities. In contrast, none of the controls and heterozygous mice showed airway occlusion. We also observed that 3 knockout mice presented 
severely asymmetric nasal cavities whereas all 12 controls $\left(8\right.$ Sned $1^{\text {WT/fl }}$ and 4 Sned $1^{f l f f}$ ) and all 7 heterozygotes had symmetrical cavities (Figure 7I, Supplementary Files 7-10).

We further analyzed variations in the morphology of the mandible using geometric morphometric analysis. PCA yielded 29 principal components, the first 5 of which represented greater than $5 \%$ of the overall variation. PC1 represented $27.38 \%$ of the variation and showed statistically significant differences across the four genotypes $(\mathrm{p}=0.009)$ (Figure 8A). A Tukey's HSD test revealed that these differences were statistically significant between the controls (Sned ${ }^{f l f l} \mathrm{p}=0.019$ ) and heterozygotes $(\mathrm{p}=0.027)$ compared to the knockout mice (Figure $8 \mathrm{~B})$. Mice clustering at the maximum extreme of variation along PC1 (PC1 max, knockout mice) showed shorter mandibular condyles (Figure 8C, lower panel), while those at the minimum extreme of variation along PC1 (PC1 min, control and heterozygous mice) tend to display longer mandibular condyles (Figure 8C, upper panel).

The comparison of the craniofacial phenotypes presented by global knockout mice versus the NCC-

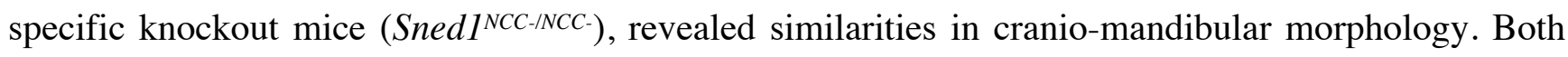
sets of knockout mice show concave nasal bridges and somewhat wider, rounder crania, along with more posteriorly positioned coronoid process, taller anterior mandibular bodies, and relatively shorter condyles. It should be noted, however, that condylar differences are more extreme in the global knockout mice, which show narrower and more foreshortened condyles than the other groups. Altogether, our results demonstrate that the deletion of Sned 1 in the NCCs partly phenocopies what we observed upon global Sned1 knockout, providing evidence that SNED1 is important for the NCC population and,

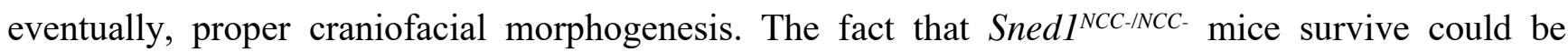
explained by the fact that SNED1 might also be acting in a cell-autonomous manner on mesodermderived structures contributing to the formation of the facial skeleton. In addition, SNED1 produced by other cell types might function as signaling cues for NCCs, ultimately ameliorating the severity of the phenotype in Sned $1^{N C C-I N C C-}$ mice.

\section{Discussion}

We report here the generation and phenotypic analysis of mouse strains in which we deleted Sned 1 globally or specifically from the neural crest cells. We showed that Snedl is essential during development, since the majority of the global knockout mice died prematurely within 2 days of birth, 
and the surviving animals exhibit severe growth defects and craniofacial malformations. Despite the broad pattern of expression of Snedl in murine embryos, the only obvious phenotypes discerned at this stage of our studies relate to overall growth, long bone length, and craniofacial features. The surviving Snedl knockout adults did not present other overt anomalies (for example, normal number of digits was counted, tongue appeared normal, complete palate closure was observed, no pigmentation defect was observed). Importantly, the phenotypes studied present variable expressivity, ranging from neonates that did not develop a lower jaw and did not survive, to the less severely affected mice that were viable and able to develop to adulthood. It is worth noting that the adult mice on which the phenotypic analyses were conducted are likely to present less severe phenotypes because they survived; yet our analyses reveal that even if mild, the phenotypic features clearly distinguished control from knockout mice. Follow-up studies, including analyses of late-stage embryos and neonates, will be required to establish the onset of craniofacial phenotype and to understand whether the variability in severity is stochastic or whether compensatory mechanisms have intervened and led to certain animals surviving whereas the majority of Snedl knockout neonates did not survive past post-natal day 2.

Based on Sned 1 expression data and the observed craniofacial malformations, we postulated that SNED1 may play a role in NCC biology. Interestingly, deletion of Snedl in the NCCs also results in growth defects and facial dysmorphic features, partly phenocopying the global knockout. We previously identified the function of SNED1 as an ECM protein promoting breast cancer metastasis..$^{5}$ Here, we report the expression of Snedl in structures that undergo EMT and cell migration during embryonic development, including the sclerotomes and the NCCs. ${ }^{56}$ The parallel has been drawn between developmental EMT and carcinoma metastasis, since in both cases, epithelial cells lose cell-cell junctions, up-regulate ECM genes such as fibronectin, remodel their adhesions to the ECM and acquire a migratory phenotype..$^{57,58}$ Therefore, the NCC-specific Snedl KO will be a useful tool to decipher which precise cellular mechanism(s) governing NCC behavior (EMT, migration, proliferation, differentiation) are controlled by SNED1. Studies of the possible roles of SNED1 in EMT of NCC may thus also further help understand how SNED1 contributes to cancer metastasis.

Once cephalic NCCs have migrated into the frontonasal region and pharyngeal arches, they need to receive signals in order to survive, proliferate, and differentiate into bone and cartilage. Future studies will also evaluate whether SNED1 contributes to any of these processes. 
As of now, neither the SNED1 receptor(s) nor the signaling pathways activated downstream of SNED1 are known. Dissecting the molecular mechanisms leading to the phenotypes described in the present study will be the focus of future investigations. In particular, integrins have been shown to be involved in different aspects of cranial neural crest cells phenotypes. ${ }^{17,59-64}$ It would thus be important to determine whether the putative integrin-binding sites found in SNED1 do indeed engage these ECM receptors, and if so, whether this is how SNED1 governs craniofacial morphogenesis. In addition, it will be crucial to identify the pathways regulating SNED1.

Of note, Snedl was among the most significantly up-regulated genes in NCC-specific Tgfbr2 knockout mice (Wnt1-Cre; Tgfbr2 $2^{f l f l}$ ) presenting abnormal palate closure (clefting) ${ }^{65}$. Although cleft palate or lip were not observed in Snedl knockout mice, this observation may suggest that SNED1 is regulated by the TGF- $\beta$ signaling pathways. Supporting this idea, Snedl was found to be enriched upon removal of $T g f b r 2$ in the developing intervertebral discs (Col2al-Cre; Tgfbr $2^{f l f f}$ ) of mouse embryos, and downregulated when sclerotome cells are treated with ligands of the TGF- $\beta$ superfamily ${ }^{66}$. The TGF- $\beta$ signaling pathway plays fundamental roles in bone and cartilage formation and homeostasis, ${ }^{67}$ but also in metastasis ${ }^{68}$ A literature search also revealed that Snedl is enriched in the mandible and frontonasal region of mouse embryos, ${ }^{49,50}$ murine differentiating osteoblasts, ${ }^{69}$ and human articular chondrocytes of fetal joints ${ }^{70}$, suggesting a putative role for SNED1 in chondrogenesis and osteogenesis in bones of the axial and appendicular skeleton. Future mechanistic investigations will benefit from the conditional Snedl knockout mouse generated in this study which will be instrumental in determining the functions of Snedl in a cell- or tissue-specific and time-specific manner.

Last, future studies of the roles of SNED1 in craniofacial development using the mouse models generated in this study may shed light on fundamental processes that, when altered, lead to craniofacial malformations in humans. ${ }^{71}$ SNED1 is localized on the long arm of chromosome 2 (2q37.3) in humans. Patients with 2q37 deletion syndrome, also described in the literature as Albright hereditary osteodystrophy-like syndrome or brachydactyly-mental retardation (BDMR) syndrome, suffer from mental retardation, facial dysmorphism, including round face and flattened nasal bridge, and skeletal abnormalities such as brachydactyly and short stature. ${ }^{72-74}$ More than 100 cases have been reported in the literature with $2 q 37$ deletion syndrome. ${ }^{75}$ Genotype-phenotype correlation studies have been performed to delineate the critical region causing BDMR.${ }^{75} H D A C 4$ has been postulated as one of the candidates responsible for BDMR. ${ }^{76}$ However, at least two cases with facial dysmorphic features have 
been reported harboring a distal 2q37.3 deletion not including HDAC476,77 (Figure 9). Since SNED1 had not been identified when most of these studies were published and that the chromosomal breakpoints had not been precisely mapped in patients diagnosed more than 20 years ago ${ }^{78,79}$ there are potentially more patients presenting with no deletion of $H D A C 4$ but with deletion of SNED1. In light of the present study, it would thus be interesting to determine to what extent the craniofacial phenotype of $2 \mathrm{q} 37.3$ deletion syndrome patients can be attributed to the loss of SNED1.

\section{Experimental Procedures}

\section{Generation of Snedl knockout mice}

All experiments involving mice were conducted in conformity with animal protocols approved by MIT's Department of Comparative Medicine and Committee on Animal Care, and by the Committee on Animal Care of the University of Illinois at Chicago in compliance with IACUC guidelines.

Two targeted Sned ${ }^{\text {tmla(KOMP)Wtsi }}$ ES cell clones (EPD0300_5_02 E02 and F01) from the C57BL/6 JM8.N4 parental ES cell line generated by the Wellcome Trust Sanger Institute ${ }^{43}$ were obtained from the KOMP Repository (www.komp.org) at UC Davis and cultured on feeder cells using the recommended media including the 2i additives MEK inhibitor PD0325901 and GSK3 inhibitor CHIR99021 (StemGent, Cambridge, MA). Three subclones of each clone were sent to Cell Line Genetics (Madison, WI) for cytogenetic analysis and two subclones of the targeted clone E02 were found to have an apparently normal mouse male karyotype. Cells from subclone E02-2D2 were microinjected into BALB/c blastocysts obtained from natural matings as previously described ${ }^{80} 10$ recipient females each received 14 embryos. 7 pregnant females gave birth to 41 pups, of which 6 showed at least $70 \%$ dark coat color against white, BALB/c fur, and allowed establishment of the Sned1 $1^{\text {tmla(KOMP)Wtsi }}$ (referred to as Sned1 $1^{\text {LacZ- }}$ ${ }^{\mathrm{Neo}}$ ) mouse line. B6.Cg-Tg(ACTFLPe)9205Dym/J (Actin-Flp; Jackson Laboratory stock No. 005703) mice allowing the ubiquitous expression of the Flp recombinase were obtained from MIT's transgenic

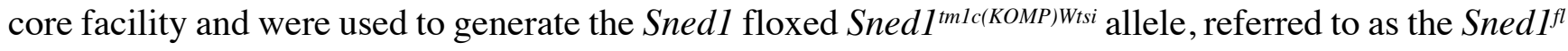
allele. B6.C-Tg(CMV-cre)1Cgn/J (CMV-Cre) mice allowing the ubiquitous expression of the Cre recombinase were purchased from Jackson Laboratory, stock No. 006054 and were used to generate the null Sned1 $1^{\text {tmld(КОмP)Wtsi }}$ allele, referred to as the Sned1-allele. Figure 2A shows a schematic view of the alleles. B6.Cg-E2f1 $1^{\mathrm{Tg}(\mathrm{Wnt1}-\mathrm{cre}) 2 \mathrm{Sor} / \mathrm{J}}$ (Wnt1-Cre2; Jackson Laboratory stock No. 022501) were crossed to 
mice carrying the Sned $l^{f l}$ allele to generate the NCC-specific Snedl knockout mouse line (Sned ${ }^{\text {NCC- }}$ allele).

The three mouse strains generated for this study have been deposited to Mutant Mouse Resource \& Research Centers (MMRRC) repository: Sned $1^{\text {LacZ-Neo }}$ strain No. 065813, Sned $1^{f l}$ strain No. 065814, and Sned1 ' strain No. 065815 and will all be made available upon publication of this manuscript.

Mice were subjected to blind visual genotyping and with a very high rate, knockout mice, whose genotype was confirmed by PCR (see below), were easily distinguished because of their craniofacial phenotype from wild-type and heterozygous littermates.

\section{Genotyping}

DNA was extracted from tail samples from 14- or 21-day old pups, or from yolk sacs or tail samples from E18.5 embryos, according to standard procedures and genotyping was performed with the following primers: Snedl (WT allele: 513 bp; floxed allele: 614 bp): Sned1_F TTCTTATTCACACCGTATGCCAGCC and Sned1_ttR CTAGTGGGCACTCATTCAGCAAACC; Null allele of Sned1 (732 bp): Sned1_F TTCTTATTCACACCGTATGCCAGCC and Sned1_R TCACATGAGCAACACGTTTGTAGG; Neo cassette (603bp): Neo_F GGGATCTCATGCTGGAGTTCTTCG and Sned1_ttR CTAGTGGGCACTCATTCAGCAAACC; LoxP site after exon 2 (374 bp): LoxP_F GAGATGGCGCAACGCAATTAATG and Sned1_R TCACATGAGCAACACGTTTGTAGGG; Flp transgene $(725 \quad b p)$ : Flp_F CACTGATATTGTAAGTAGTTTGC and Flp_R CTAGTGCGAAGTAGTGATCAGG; Cre transgene (700 bp): Cre_F TGCTGTACTGGTTATGCGG and Cre_R TTGCCCCTGTTTCACTATCCAG.

PCR amplification was performed using the DreamTaq DNA polymerase (Thermo Fisher Scientific, Waltham, MA). Cycling conditions were: $95^{\circ} \mathrm{C}$ for 2 min.; 35 cycles at: $95^{\circ} \mathrm{C}$ for $30 \mathrm{sec}$., $60^{\circ} \mathrm{C}$ for 30 sec., $72^{\circ} \mathrm{C}$ for $45 \mathrm{sec}$.; final extension at $72^{\circ} \mathrm{C}$ for $3 \mathrm{~min}$. Representative genotyping gels are presented in Figure 2B and Figure 7A. Fragments amplified with primers Sned1_F and Sned1_ttR corresponding to the Sned $1^{\text {LacZ-Neo }}$ allele $(7518 \mathrm{bp})$ and the Sned $1^{\text {WT }}$ allele of the null mice $(2113 \mathrm{bp})$ are not observed in Figure 2B since the PCR extension step was optimized to amplify shorter fragments.

\section{Survival analysis}


Goodness-of-fit analysis was performed using a two-tailed chi-square $\left(\chi^{2}\right)$ test (GraphPad). This statistical analysis compares expected (Mendelian distribution) and observed numbers of embryos (E18.5) or pups (p21) to determine whether survival correlates with animal genotypes.

\section{Isolation of mouse embryonic fibroblasts and quantitative PCR}

Mouse embryonic fibroblasts (MEFs) were isolated from E13.5 to E15.5 embryos obtained by timed mating of heterozygous Sned $1^{\text {WT/LacZ-Neo }}$ mice. For quantitative PCR (qPCR), RNA was isolated from the cells using the RNeasy kit (Qiagen, Germantown, MD) and cDNA was synthesized by reverse transcription using the First-Strand cDNA Synthesis Kit (Promega, Madison, WI). qPCR reactions were performed using Bio-Rad SYBR Green Supermix (Bio-Rad, Hercules, CA) according to the manufacturer's instructions. PCR conditions were $95^{\circ} \mathrm{C}$ for 10 minutes, followed by 40 cycles of $95^{\circ} \mathrm{C}$ for 20 seconds, $58^{\circ} \mathrm{C}$ for 30 seconds, and $72^{\circ} \mathrm{C}$ for 30 seconds. $\mathrm{qPCR}$ data analysis was performed using

Bio-Rad CFX Manager Software. The following primers were used: Actin_F TGtatgaAGGCTTtGGtCTCC; Actin_R GTCTCAAGTCAGTGTACAGGC; Sned1_F GTAGATGGAAGAGGAAGAGTGAG; Sned1_R CTGTTCTTGGGTAGCTGGAG.

\section{Riboprobe synthesis and in-situ hybridization}

Murine Snedl cDNA was amplified by PCR using the following primers: forward: 5'GGACTAGTATGCGCCTCGGC-3' and Reverse 5'-CCCAAGCTTGCACTGGGGAGGC-3', covering the 5' end half of Sned1 cDNA to ensure detection of the mRNA encoding secreted SNED1. PCR products were subcloned into pBlueScript KS+ plasmid using SpeI and HindIII restriction sites. Digoxigenin-labeled sense and antisense riboprobes of Snedl were synthesized with T7 and T3 RNA polymerases (Roche, Basel, Switzerland), respectively.

Deskinned E18.5 mouse embryos were fixed in $10 \%$ neutral-buffered formalin overnight at $4^{\circ} \mathrm{C}$, embedded in paraffin and sectioned. Sections were dewaxed, rehydrated, and permeabilized with 20 $\mu \mathrm{g} / \mathrm{ml}$ Proteinase K. Sections were incubated with pre-warmed hybridization solution (50\% formamide, 5X SSC, $1 \%$ SDS, $10 \mu \mathrm{g} / \mathrm{ml}$ tRNA, $10 \mu \mathrm{g} / \mathrm{ml}$ heparin) for 1 hour at $60^{\circ} \mathrm{C}$. Hybridization with Snedl riboprobes $(50 \mathrm{ng} / \mathrm{ml})$ was performed overnight at $65^{\circ} \mathrm{C}$ followed by incubation with a-Digoxigenin-AP antibody (1:2000; Roche, Basel, Switzerland) for 2 hours. Colorimetric reaction was developed using BM-Purple for 3 days at $4^{\circ} \mathrm{C}$. Sample were imaged using an Axio Imager.Z2 and an Axiocam 105 color camera (Zeiss). 


\section{$\beta$-galactosidase assay}

$\beta$-galactosidase assay (LacZ staining) was performed on whole heterozygous Sned1 ${ }^{\text {WT/LacZ-Neo }}$ embryos (E11.5 and E13.5) according to previously published detailed protocols. ${ }^{81,82}$ For visualization of wholemount staining, embryos were cleared by incubation in solutions of increasing glycerol and decreasing potassium hydroxide concentrations following a published protocol. ${ }^{83}$ LacZ-stained embryos were embedded in paraffin and sectioned, sections were then dewaxed and rehydrated, and hematoxylin and eosin counter-staining was performed following standard procedures.

\section{Microcomputed tomography image acquisition and analysis}

An eXplore CT $120 \mu$ CT system (Northridge Tri-Modality Imaging Inc., Chatsworth, CA) was used for in-vivo imaging of a cohort of 19 adult (6-month-old) and 22 (p0.5) global Sned1 knockout animals and control littermates. Mice were imaged under anesthesia (induced at 3\% isoflurane in oxygen, maintained at between 2-2.5\% during imaging) in groups of 4 in a custom mouse holder. Scanner settings were as follows: 720 views, 360 -degree rotation, $70 \mathrm{kVp}, 50 \mathrm{~mA}, 32 \mathrm{~ms}$ integration time with $2 \times 2$ detector pixel binning (isotropic nominal resolution of 50 microns). Data were reconstructed using the Parallax Innovations (Ilderton, ON, Canada) GPU accelerated reconstruction engine for the eXplore CT120.

A cohort of 30 adult (7 to 15-month-old) NCC-specific Sned1 knockout animals and control littermates were scanned using a custom-built GE phoenix v|tome|x s240 dual-tube X-ray $\mu \mathrm{CT}$ system (General Electric, Boston, MA) located at the University of Chicago. Data was acquired using a high power 240 $\mathrm{kV}$ tube. Scanner settings were as follows: 1,000 views, 360-degree rotation, $60 \mathrm{kVp}, 350 \mu \mathrm{A}, 250 \mathrm{~ms}$ integration time to achieve isotropic reconstructions with resolution (voxel size) of 40 microns. Data were reconstructed using the datos|x reconstruction software (General Electric, Boston, MA). The same parameter of beam hardening correction was applied to all samples. Reconstructed $\mu \mathrm{CT}$ scans of the mouse crania were converted to TIFF files and imported into 3D Slicer or ImageJ for further analysis.

\section{Measurement of long bone length}

$3 \mathrm{D}$ volume renderings of long bones were created from $\mu \mathrm{CT}$ scan files in 3D Slicer. Length of long bones was measured in $\mathrm{mm}$. Unpaired t-tests were conducted to analyze differences in the length of long bones between genotype groups.

\section{Measurement of cranial suture length}


Variations in the cranial sutures were visually observed in 3D Slicer. ${ }^{84,85}$ The nasal (anterior tip of nasal bones to premaxillary suture), frontal (premaxillary suture to coronal suture), and sagittal (coronal to interparietal suture) sutures were observed through the transverse plane and were measured anteroposteriorly (in $\mathrm{mm}$ ), while the coronal sutures were observed through the sagittal plane. Unpaired t-tests were conducted to analyze differences in the length of the calvarial sutures between genotype groups.

\section{Assessment of nasal cavity symmetry and occlusion}

Analyses were conducted using ImageJ software, using the "Multi VFF Opener" plug-in to open the $\mu \mathrm{CT}$ scan files. Variations in nasal cavity symmetry and occlusion were visually observed in 3D Slicer ${ }^{84,85}$ and scored.

\section{Landmarking and geometric morphometric analysis of the skull}

We selected a total of 30 landmarks (22 traditional coordinate landmarks, 8 sliding semi-landmarks) which were deemed to represent well overall cranial morphology (see positioning of landmarks in Supplementary Method 1). The 8 sliding semi-landmarks were positioned along the nasal suture, as visual observation indicated that nasal and mid-facial morphology may be particularly affected in these mice. To undertake complex phenotyping, we used standard geometric morphometric (GM) methodologies for analyzing 3D shapes. First, a generalized Procrustes analysis (GPA) ${ }^{86}$ was run on the raw coordinate landmark data using the Geomorph package in $\mathrm{R} .{ }^{87}$ This procedure scales, rotates, and translates landmark data to align them for further analysis. Importantly, this step (through scaling) reduces size to a separate variable so that shape can be studied in isolation. ${ }^{88}$ Semi-landmarks were slid, minimizing bending energy, as a part of the GPA. Next, to investigate shape variation in the cohort, we ran a principal component analysis (PCA) on the GPA data. Statistical significance of each of the first four PCs was assessed using a one-way non-parametric Kruskal-Wallis analysis of variance (ANOVA) by genotype followed by a Tukey's HSD test. All analyses were performed on both the Sned1LacZ-Neo and Sned $1^{\text {NCC- }}$ transgenic line datasets, separately.

\section{Landmarking and geometric morphometric analysis of the mandible}

We selected a total of 18 traditional landmarks on the right side of the mandible which were deemed to represent well overall mandibular morphology (see positioning of landmarks in Supplementary Method $2)^{45}$. Next, to investigate shape variation in the cohort, we ran a PCA on the GPA data using the R package Geomorph. ${ }^{87}$ Statistical significance of each of the first seven PCs was assessed using a one- 
way non-parametric Kruskal-Wallis ANOVA by genotype followed by a Tukey's HSD test. All analyses were performed on both the Sned $1^{\text {LacZ-Neo }}$ and Sned $1^{\text {NCC- }}$ transgenic line datasets, separately. 


\section{Acknowledgements}

The authors wish to acknowledge Dr. Aurora Burds Connor and Noranne Enzer from the ES Cell and Transgenics Facility (MIT) for their help generating the Snedl mouse lines, Lisa Billings and Katherine De La Hoz from the MIT Department of Comparative Medicine for their assistance with mouse husbandry, Kathleen Cormier from The Hope Babette Tang (1983) Histology Facility (MIT) for her help with preparation and sectioning of histological samples, Milton Cornwall-Brady and Dr. Scott Malstrom from the Animal Imaging \& Preclinical Testing Core Facility (MIT), and April I. Neander (University of Chicago) for their assistance with $\mu \mathrm{CT}$ image acquisition. The authors also wish to acknowledge Dr. Patrick Murphy (Hynes lab) for noticing initially the craniofacial anomalies of the Snedl knockout mice, Ying Huang and Zhigang Jiang (Hynes lab) for assistance with genotyping, Martin Davis (Naba lab) for assistance with genotyping and mouse colony maintenance, Dr. Naiche Adler for technical assistance with in-situ hybridization, and Ali Thahab (Naba lab) for his assistance with the analysis of human clinical data.

The Sned1 mouse strains used for this research project were created from ES cell clone EPD0300_5_E02, obtained from the KOMP Repository (www.komp.org) and generated by the Wellcome Trust Sanger Institute. Targeting vectors used were generated by the Wellcome Trust Sanger Institute and the Children's Hospital Oakland Research Institute as part of the Knockout Mouse Project (3U01HG004080). 


\section{References}

1. Hynes RO, Naba A. Overview of the matrisome - An inventory of extracellular matrix constituents and functions. Cold Spring Harb Perspect Biol. 2012;4(1):a004903. doi:10.1101/cshperspect.a004903

2. Rozario T, DeSimone DW. The Extracellular Matrix In Development and Morphogenesis: A Dynamic View. Dev Biol. 2010;341(1):126-140. doi:10.1016/j.ydbio.2009.10.026

3. Bonnans C, Chou J, Werb Z. Remodelling the extracellular matrix in development and disease. Nat Rev Mol Cell Biol. 2014;15(12):786-801. doi:10.1038/nrm3904

4. Bateman JF, Boot-Handford RP, Lamandé SR. Genetic diseases of connective tissues: cellular and extracellular effects of ECM mutations. Nat Rev Genet. 2009;10(3):173-183. doi:10.1038/nrg2520

5. Naba A, Clauser KR, Lamar JM, Carr SA, Hynes RO. Extracellular matrix signatures of human mammary carcinoma identify novel metastasis promoters. eLife. 2014;3. doi:10.7554/eLife.01308

6. Pickup MW, Mouw JK, Weaver VM. The extracellular matrix modulates the hallmarks of cancer. EMBO Rep. 2014;15(12):1243-1253. doi:10.15252/embr.201439246

7. DeSimone DW, Mecham RP, eds. Extracellular Matrix in Development. 1st ed. Springer; 2013. doi:10.1007/978-3-642-35935-4

8. Czirok A, Rongish BJ, Little CD. Extracellular Matrix Dynamics in Early Development. In: DeSimone DW, Mecham RP, eds. Extracellular Matrix in Development. 1st ed. Biology of Extracellular Matrix. Springer; 2013:19-36. doi:10.1007/978-3-642-35935-4_2

9. George EL, Hynes RO. Gene targeting and generation of mutant mice for studies of cellextracellular matrix interactions. Methods Enzymol. 1994;245:386-420. doi:10.1016/0076$6879(94) 45021-8$

10. Miner JH. Extracellular Matrix in Development and Disease. 1st ed. Elsevier; 2005. Accessed May 13, 2020. https://www.elsevier.com/books/extracellular-matrix-in-development-anddisease/unknown/978-0-444-51846-0

11. Cruz Walma DA, Yamada KM. The extracellular matrix in development. Development. 2020;147(10). doi:10.1242/dev.175596

12. Arikawa-Hirasawa E, Watanabe H, Takami H, Hassell JR, Yamada Y. Perlecan is essential for cartilage and cephalic development. Nat Genet. 1999;23(3):354-358. doi:10.1038/15537

13. Smyth N, Vatansever HS, Murray P, et al. Absence of Basement Membranes after Targeting the LAMC1 Gene Results in Embryonic Lethality Due to Failure of Endoderm Differentiation. $J$ Cell Biol. 1999;144(1):151-160. doi:10.1083/jcb.144.1.151

14. Alpy F, Jivkov I, Sorokin L, et al. Generation of a conditionally null allele of the laminin $\alpha 1$ gene. genesis. 2005;43(2):59-70. doi:10.1002/gene.20154 
15. George EL, Georges-Labouesse EN, Patel-King RS, Rayburn H, Hynes RO. Defects in mesoderm, neural tube and vascular development in mouse embryos lacking fibronectin. Development. 1993;119(4):1079-1091.

16. Stephens LE, Sutherland AE, Klimanskaya IV, et al. Deletion of beta 1 integrins in mice results in inner cell mass failure and peri-implantation lethality. Genes Dev. 1995;9(15):1883-1895. doi:10.1101/gad.9.15.1883

17. Bökel C, Brown NH. Integrins in Development: Moving on, Responding to, and Sticking to the Extracellular Matrix. Dev Cell. 2002;3(3):311-321. doi:10.1016/S1534-5807(02)00265-4

18. Lallier T, Leblanc G, Artinger KB, Bronner-Fraser M. Cranial and trunk neural crest cells use different mechanisms for attachment to extracellular matrices. Development. 1992;116(3):531-541.

19. Perris R, Perissinotto D. Role of the extracellular matrix during neural crest cell migration. Mech Dev. 2000;95(1-2):3-21. doi:10.1016/S0925-4773(00)00365-8

20. Tucker RP. Abnormal neural crest cell migration after the in vivo knockdown of tenascin-C expression with morpholino antisense oligonucleotides. Dev Dyn. 2001;222(1):115-119. doi:10.1002/dvdy.1171

21. Perissinotto D, Iacopetti P, Bellina I, et al. Avian neural crest cell migration is diversely regulated by the two major hyaluronan-binding proteoglycans $\mathrm{PG}-\mathrm{M} /$ versican and aggrecan. Development. 2000;127(13):2823-2842.

22. Shinohara Y, Okamoto K, Goh Y, Kiga N, Tojyo I, Fujita S. Inhibition of Fibrous Adhesion Formation in the Temporomandibular Joint of Tenascin-C Knockout Mice. Eur J Histochem EJH. 2014;58(4). doi:10.4081/ejh.2014.2337

23. Embree MC, Kilts TM, Ono M, et al. Biglycan and Fibromodulin Have Essential Roles in Regulating Chondrogenesis and Extracellular Matrix Turnover in Temporomandibular Joint Osteoarthritis. Am J Pathol. 2010;176(2):812-826. doi:10.2353/ajpath.2010.090450

24. Manocha S, Farokhnia N, Khosropanah S, Bertol JW, Santiago J, Fakhouri WD. Systematic review of hormonal and genetic factors involved in the nonsyndromic disorders of the lower jaw. Dev Dyn. 2019;248(2):162-172. doi:10.1002/dvdy.8

25. Leimeister C, Schumacher N, Diez H, Gessler M. Cloning and expression analysis of the mouse stroma marker Snep encoding a novel nidogen domain protein. Dev Dyn. 2004;230(2):371-377. doi:10.1002/dvdy.20056

26. Longati $\mathrm{P}$, Jia $\mathrm{X}$, Eimer J, et al. 3D pancreatic carcinoma spheroids induce a matrix-rich, chemoresistant phenotype offering a better model for drug testing. BMC Cancer. 2013;13:95. doi:10.1186/1471-2407-13-95

27. Weissmueller S, Manchado E, Saborowski M, et al. Mutant p53 drives pancreatic cancer metastasis through cell-autonomous PDGF receptor $\beta$ signaling. Cell. 2014;157(2):382-394. doi:10.1016/j.cell.2014.01.066 
28. Le Lièvre CS. Role of mesectodermal cells arising from the cephalic neural crest in the formation of the branchial arches and visceral skeleton. J Embryol Exp Morphol. 1974;31(2):453-477.

29. Le Lièvre CS, Le Douarin NM. Mesenchymal derivatives of the neural crest: analysis of chimaeric quail and chick embryos. J Embryol Exp Morphol. 1975;34(1):125-154.

30. Chai Y, Jiang $\mathrm{X}$, Ito $\mathrm{Y}$, et al. Fate of the mammalian cranial neural crest during tooth and mandibular morphogenesis. Development. 2000;127(8):1671-1679.

31. Noden DM. The role of the neural crest in patterning of avian cranial skeletal, connective, and muscle tissues. Dev Biol. 1983;96(1):144-165. doi:10.1016/0012-1606(83)90318-4

32. Hohenester E, Engel J. Domain structure and organisation in extracellular matrix proteins. Matrix Biol. 2002;21(2):115-128. doi:10.1016/s0945-053x(01)00191-3

33. Naba A, Clauser KR, Hoersch S, Liu H, Carr SA, Hynes RO. The Matrisome: In Silico Definition and In Vivo Characterization by Proteomics of Normal and Tumor Extracellular Matrices. Mol Cell Proteomics. 2012;11(4):M111.014647. doi:10.1074/mcp.M111.014647

34. Letunic I, Doerks T, Bork P. SMART: recent updates, new developments and status in 2015. Nucleic Acids Res. 2015;43(Database issue):D257-D260. doi:10.1093/nar/gku949

35. Finn RD, Attwood TK, Babbitt PC, et al. InterPro in 2017-beyond protein family and domain annotations. Nucleic Acids Res. 2017;45(D1):D190-D199. doi:10.1093/nar/gkw1107

36. Ozbek S, Balasubramanian PG, Chiquet-Ehrismann R, Tucker RP, Adams JC. The evolution of extracellular matrix. Mol Biol Cell. 2010;21(24):4300-4305. doi:10.1091/mbc.E10-03-0251

37. Huxley-Jones J, Pinney JW, Archer J, Robertson DL, Boot-Handford RP. Back to basics - How the evolution of the extracellular matrix underpinned vertebrate evolution. Int $J$ Exp Pathol. 2009;90(2):95-100. doi:10.1111/j.1365-2613.2008.00637.x

38. Hynes RO. The evolution of metazoan extracellular matrix. J Cell Biol. 2012;196(6):671-679. doi:10.1083/jcb.201109041

39. Bronner ME, LeDouarin NM. Evolution and Development of the Neural Crest: An Overview. Dev Biol. 2012;366(1):2-9. doi:10.1016/j.ydbio.2011.12.042

40. Muñoz WA, Trainor PA. Neural crest cell evolution: how and when did a neural crest cell become a neural crest cell. Curr Top Dev Biol. 2015;111:3-26. doi:10.1016/bs.ctdb.2014.11.001

41. Ruoslahti E. Rgd and Other Recognition Sequences for Integrins. Annu Rev Cell Dev Biol. 1996;12(1):697-715. doi:10.1146/annurev.cellbio.12.1.697

42. Campbell ID, Humphries MJ. Integrin Structure, Activation, and Interactions. Cold Spring Harb Perspect Biol. 2011;3(3). doi:10.1101/cshperspect.a004994

43. Skarnes WC, Rosen B, West AP, et al. A conditional knockout resource for the genome-wide study of mouse gene function. Nature. 2011;474(7351):337-342. doi:10.1038/nature10163 
44. Cooke SB, Terhune CE. Form, Function, and Geometric Morphometrics: Form, Function, and Geometric Morphometrics. Anat Rec. 2015;298(1):5-28. doi:10.1002/ar.23065

45. Martínez-Vargas J, Muñoz-Muñoz F, Martinez-Maza C, Molinero A, Ventura J. Postnatal mandible growth in wild and laboratory mice: Differences revealed from bone remodeling patterns and geometric morphometrics. J Morphol. 2017;278(8):1058-1074. doi:10.1002/jmor.20694

46. Douarin L. The Neural Crest. Cambridge University Press; 1982.

47. Dash S, Trainor PA. The development, patterning and evolution of neural crest cell differentiation into cartilage and bone. Bone. 2020;137:115409. doi:10.1016/j.bone.2020.115409

48. Dasgupta K, Chung JU, Asam K, Jeong J. Molecular patterning of the embryonic cranial mesenchyme revealed by genome-wide transcriptional profiling. Dev Biol. 2019;455(2):434-448. doi:10.1016/j.ydbio.2019.07.015

49. Feng W, Leach SM, Tipney H, et al. Spatial and Temporal Analysis of Gene Expression during Growth and Fusion of the Mouse Facial Prominences. Bridger JM, ed. PLoS ONE. 2009;4(12):e8066. doi:10.1371/journal.pone.0008066

50. Tipney HJ, Leach SM, Feng W, Spritz R, Williams T, Hunter L. Leveraging existing biological knowledge in the identification of candidate genes for facial dysmorphology. BMC Bioinformatics. 2009;10 Suppl 2:S12. doi:10.1186/1471-2105-10-S2-S12

51. Couly GF, Coltey PM, Douarin NML. The triple origin of skull in higher vertebrates: a study in quail-chick chimeras. Development. 1993;117(2):409-429.

52. Noden DM, Trainor PA. Relations and interactions between cranial mesoderm and neural crest populations. J Anat. 2005;207(5):575-601. doi:10.1111/j.1469-7580.2005.00473.x

53. Jiang X, Iseki S, Maxson RE, Sucov HM, Morriss-Kay GM. Tissue origins and interactions in the mammalian skull vault. Dev Biol. 2002;241(1):106-116. doi:10.1006/dbio.2001.0487

54. Trainor PA, Melton KR, Manzanares M. Origins and plasticity of neural crest cells and their roles in jaw and craniofacial evolution. Int J Dev Biol. 2003;47(7-8):541-553.

55. Lewis AE, Vasudevan HN, O’Neill AK, Soriano P, Bush JO. The widely used Wnt1-Cre transgene causes developmental phenotypes by ectopic activation of Wnt signaling. Dev Biol. 2013;379(2):229-234. doi:10.1016/j.ydbio.2013.04.026

56. Thiery JP, Acloque H, Huang RYJ, Nieto MA. Epithelial-Mesenchymal Transitions in Development and Disease. Cell. 2009;139(5):871-890. doi:10.1016/j.cell.2009.11.007

57. Gallik KL, Treffy RW, Nacke LM, et al. Neural crest and cancer: Divergent travelers on similar paths. Mech Dev. 2017;148:89-99. doi:10.1016/j.mod.2017.08.002

58. Brabletz T, Kalluri R, Nieto MA, Weinberg RA. EMT in cancer. Nat Rev Cancer. 2018;18(2):128134. doi:10.1038/nrc.2017.118 
59. McKeown SJ, Wallace AS, Anderson RB. Expression and function of cell adhesion molecules during neural crest migration. Dev Biol. 2013;373(2):244-257. doi:10.1016/j.ydbio.2012.10.028

60. Pietri T, Thiery JP, Dufour S. Differential expression of $\beta 3$ integrin gene in chick and mouse cranial neural crest cells. Dev Dyn. 2003;227(2):309-313. doi:10.1002/dvdy.10299

61. Thiery JP. Cell adhesion in development: a complex signaling network. Curr Opin Genet Dev. 2003;13(4):365-371. doi:10.1016/s0959-437x(03)00088-1

62. Turner CJ, Badu-Nkansah K, Crowley D, Flier A van der, Hynes RO. $\alpha 5$ and $\alpha$ v integrins cooperate to regulate vascular smooth muscle and neural crest functions in vivo. Development. 2015;142(4):797-808. doi:10.1242/dev.117572

63. Liang D, Wang X, Mittal A, et al. Mesodermal expression of integrin $\alpha 5 \beta 1$ regulates neural crest development and cardiovascular morphogenesis. Dev Biol. 2014;395(2):232-244. doi:10.1016/j.ydbio.2014.09.014

64. Wang X, Astrof S. Neural crest cell-autonomous roles of fibronectin in cardiovascular development. Development. 2016;143(1):88-100. doi:10.1242/dev.125286

65. Pelikan RC, Iwata J, Suzuki A, Chai Y, Hacia JG. Identification of Candidate Downstream Targets of TGF $\beta$ Signaling During Palate Development by Genome-Wide Transcript Profiling. J Cell Biochem. 2013;114(4):796-807. doi:10.1002/jcb.24417

66. Sohn P, Cox M, Chen D, Serra R. Molecular profiling of the developing mouse axial skeleton: a role for Tgfbr2 in the development of the intervertebral disc. BMC Dev Biol. 2010;10:29. doi:10.1186/1471-213X-10-29

67. Wu M, Chen G, Li Y-P. TGF- $\beta$ and BMP signaling in osteoblast, skeletal development, and bone formation, homeostasis and disease. Bone Res. 2016;4(1):1-21. doi:10.1038/boneres.2016.9

68. Padua D, Massagué J. Roles of TGFbeta in metastasis. Cell Res. 2009;19(1):89-102. doi:10.1038/cr.2008.316

69. Cray Jr JJ, Khaksarfard K, Weinberg SM, Elsalanty M, Yu JC. Effects of Thyroxine Exposure on Osteogenesis in Mouse Calvarial Pre-Osteoblasts. PLOS ONE. 2013;8(7):e69067. doi:10.1371/journal.pone.0069067

70. $\mathrm{Wu} \mathrm{L}$, Bluguermann $\mathrm{C}$, Kyupelyan L, et al. Human developmental chondrogenesis as a basis for engineering chondrocytes from pluripotent stem cells. Stem Cell Rep. 2013;1(6):575-589. doi:10.1016/j.stemcr.2013.10.012

71. Van Otterloo E, Williams T, Artinger KB. The old and new face of craniofacial research: How animal models inform human craniofacial genetic and clinical data. Dev Biol. 2016;415(2):171187. doi:10.1016/j.ydbio.2016.01.017

72. Online Mendelian Inheritance in Man, OMIM ${ }^{\circledR}$. Johns Hopkins University, Baltimore, MD. MIM Number: \#600430: 09/25/2015. World Wide Web URL: https://omim.org/. 
73. Aldred MA, Sanford ROC, Thomas NS, et al. Molecular analysis of 20 patients with $2 \mathrm{q} 37.3$ monosomy: definition of minimum deletion intervals for key phenotypes. $J$ Med Genet. 2004;41(6):433-439. doi:10.1136/jmg.2003.017202

74. Shrimpton AE, Braddock BR, Thomson LL, Stein CK, Hoo JJ. Molecular delineation of deletions on 2q37.3 in three cases with an Albright hereditary osteodystrophy-like phenotype. Clin Genet. 2004;66(6):537-544. doi:10.1111/j.1399-0004.2004.00363.x

75. Le TN, Williams SR, Alaimo JT, Elsea SH. Genotype and phenotype correlation in 103 individuals with $2 \mathrm{q} 37$ deletion syndrome reveals incomplete penetrance and supports HDAC4 as the primary genetic contributor. Am J Med Genet A. 2019;179(5):782-791. doi:10.1002/ajmg.a.61089

76. Williams SR, Aldred MA, Der Kaloustian VM, et al. Haploinsufficiency of HDAC4 causes brachydactyly mental retardation syndrome, with brachydactyly type E, developmental delays, and behavioral problems. Am J Hum Genet. 2010;87(2):219-228. doi:10.1016/j.ajhg.2010.07.011

77. Leroy $\mathrm{C}$, Landais $\mathrm{E}$, Briault $\mathrm{S}$, et al. The 2q37-deletion syndrome: an update of the clinical spectrum including overweight, brachydactyly and behavioural features in 14 new patients. Eur J Hum Genet. 2013;21(6):602-612. doi:10.1038/ejhg.2012.230

78. Wilson LC, Leverton K, Luttikhuis MEMO, et al. Brachydactyly and Mental Retardation: An Albright Hereditary Osteodystrophy-like Syndrome Localized to 2q37. Am J Hum Genet. 1995;56(2):400-407.

79. Power MM, James RS, Barber JC, et al. RDCI, the vasoactive intestinal peptide receptor: a candidate gene for the features of Albright hereditary osteodystrophy associated with deletion of 2q37. J Med Genet. 1997;34(4):287-290. doi:10.1136/jmg.34.4.287

80. Esmail MY, Qi P, Connor AB, Fox JG, García A. Generating Chimeric Mice by Using Embryos from Nonsuperovulated BALB/c Mice Compared with Superovulated BALB/c and Albino C57BL/6 Mice. J Am Assoc Lab Anim Sci JAALAS. 2016;55(4):400-405.

81. Burn SF. Detection of $\beta$-galactosidase activity: X-gal staining. Methods Mol Biol Clifton NJ. 2012;886:241-250. doi:10.1007/978-1-61779-851-1_21

82. Gierut JJ, Jacks TE, Haigis KM. Whole-mount X-Gal staining of mouse tissues. Cold Spring Harb Protoc. 2014;2014(4):417-419. doi:10.1101/pdb.prot073452

83. Schatz O, Golenser E, Ben-Arie N. Clearing and photography of whole mount X-gal stained mouse embryos. BioTechniques. 2005;39(5):650, 652, 654 passim. doi:10.2144/000112034

84. Fedorov A, Beichel R, Kalpathy-Cramer J, et al. 3D Slicer as an Image Computing Platform for the Quantitative Imaging Network. Magn Reson Imaging. 2012;30(9):1323-1341. doi:10.1016/j.mri.2012.05.001

85. Pieper S, Halle M, Kikinis R. 3D SLICER. In: Proceedings of the 1st IEEE International Symposium on Biomedical Imaging: From Nano to Macro 2004. Vol 1. IEEE International Symposium on Biomedical Imaging ISBI 2004; 2004:632:635. http://www.spl.harvard.edu/publications/item/view/91 
86. Goodall C. Procrustes Methods in the Statistical Analysis of Shape. J R Stat Soc Ser B Methodol. 1991;53(2):285-339.

87. Adams D, Collyer M, Kaliontzopoulou A. Geomorph: Geometric Morphometric Analyses of 2D/3D Landmark Data.; 2020. Accessed September 3, 2020. https://CRAN.Rproject.org $/$ package $=$ geomorph

88. Zelditch ML, Swiderski DL, Sheets HD. Geometric Morphometrics for Biologists: A Primer. 2nd ed. Elsevier; 2012. Accessed May 12, 2020. https://www.elsevier.com/books/geometricmorphometrics-for-biologists/zelditch/978-0-12-386903-6 


\section{Figure Legends}

\section{Figure 1. SNED1 phylogeny}

Schematic representation of the protein domains of SNED1 and alignment between the human $(H s)$ sequence of SNED1 (UniProt accession Q8TER0) and orthologs found in mouse (M. musculus, Mm; UniProt accession Q70E20); rat (R. Norvegicus, Rn; UniProt accession Q5ZQU0); chicken (G. gallus, Gg; UniProt accession A0A1D5P671); zebrafish (D. rerio, Dr; UniProt accession E7F2S5); and frog (X. laevis, $\mathrm{Xl}$; UniProt accession A0A1L8GAZ2). Alignment was performed using Protein BLAST (https://blast.ncbi.nlm.nih.gov/Blast.cgi?PAGE=Proteins). Percent identity and percent homology are indicated for each domain or fragment of SNED1 and for the full-length proteins. Protein length in amino acid (aa) is also indicated.

\section{Figure 2. Snedl knockout strategies and genotyping}

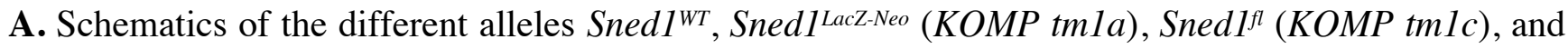
Sned l- (KOMP tmld). Arrows indicate primers used for genotyping.

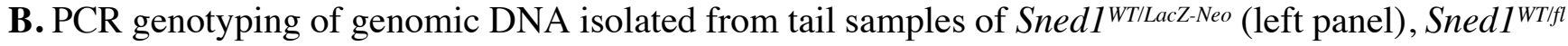
(middle panel), and Sned $1^{W T /}$ (right panel) mice.

C. RT-qPCR was used to monitor the level of expression of Snedl in mouse embryonic fibroblasts isolated from Snedl ${ }^{\text {WT/WT }}(\mathrm{n}=1)$, Sned $1^{\text {WTLacZ-Neo }}(\mathrm{n}=3)$, or Sned $1^{\text {LacZ-NeolLacZ-Neo }}(\mathrm{n}=3)$ embryos. Bar charts represent normalized expression of Snedl relative to wild-type cells. Actin expression level was used to normalize RT-qPCR data. Data are presented as means \pm S.E.M.

D. In-situ hybridization with digoxigenin-labeled Sned 1 sense riboprobe (negative control) or antisense riboprobe of sagittal sections from Sned $1^{W T / W T}$, Sned $1^{W T /}$, or Sned $1^{-\digamma}$ E18.5 mouse embryos. Scale bar represents $100 \mu \mathrm{m}$. c: costal cartilage.

\section{Figure 3. Surviving Sned1 knockout mice present growth defects}

A. Representative pictures of heterozygous (left) and knockout (right) 21-day-old mice illustrate the smaller size of surviving knockout mice.

B. Dotplots represent the weight of 14-day-old pups of the LacZ-Neo transgenic line. Numbers of mice per genotype are indicated. Line represents average weight of the genotype group. Statistical analysis was performed using an unpaired t-test. 
C. Dotplots represent the weight of p0.5 neonates of the LacZ-Neo transgenic line. Numbers of mice per genotype are indicated. Line represents average weight of the genotype group. Statistical analysis was performed using an unpaired t-test.

D. Dotplots represent the weight of 14-day-old pups of the null transgenic line. Numbers of mice per genotype are indicated. Line represents average weight of the genotype group. Statistical analysis was performed using an unpaired t-test.

E-F. Dotplots represent the length of the humerus (E) and femur (F) of 19 adult (6-month-old) mice of the LacZ-Neo transgenic line. Numbers of mice per genotype are indicated. Line represents average weight of the genotype group. Statistical analysis was performed using an unpaired t-test.

\section{Figure 4. Sned1 knockout results in craniofacial malformations}

A-F. Examination of the skull of a cohort of 19 adult (6-month-old) of the LacZ-Neo transgenic line.

A. $\mu \mathrm{CT}$ scans showing variation in skull morphology based upon genotype. Note the nasal bridge

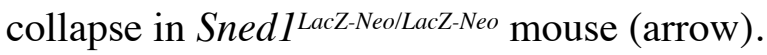

B. Nasal (left panel) and frontal (right panel) suture length. Numbers of mice per genotype are indicated. Line represents average length of the genotype group. Statistical analysis was performed using an unpaired t-test.

C. Scatterplot of PC1 and PC2 for variation in the mouse cranial morphology. Statistical analysis was performed using a one-way non-parametric Kruskal-Wallis ANOVA.

D. Boxplot of cranial PC1 data by mouse genotype. Numbers of mice per genotype are indicated. Statistical analysis was performed using a Tukey's HSD test.

E. Wireframes depicting mouse cranium side (left) and top (right) views and variation of the shape as a function of genotypes. Grey dots represent the consensus cranium shape, black dots represent the shape

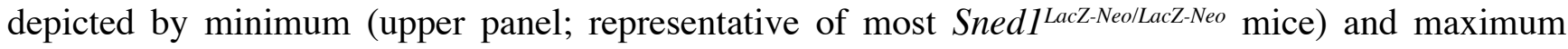
(lower panel; representative of most Sned $1^{W T W T}$ and Sned1 $1^{\text {WTLacz-Neo }}$ mice) PC1 range. C: caudal; R: rostral.

F. Occlusion and asymmetry assessment of the nasal cavity of adult mice. Arrow indicates occlusion and arrowheads indicate asymmetries. See also Supplementary Files 1-3.

G. Occlusion and asymmetry assessment of the nasal cavity of $22 \mathrm{p} 0.5$ neonates. Arrow indicates occlusion. See also Supplementary Files 4-6. 


\section{Figure 5. Sned1 knockout results in under-developed mandible}

A. Representative litter of p0.5 neonates. Black arrows indicate the presence of milk the stomach of wild-type and heterozygous mice. Note the absence of milk in the stomach of Snedl LacZ-Neo/LacZ-Neo neonates. Blue arrow points to the tails of knockout neonates which fail to curl, perhaps related to the strong expression in tail-bud somites and mesoderm (Figure 6).

B-D. Examination of the mandible of a cohort of 19 adult (6-month-old) global Snedl knockout animals and control littermates.

B. Scatterplot of PC1 and PC2 for variation in the mouse mandibular morphology. Statistical analysis was performed using a one-way non-parametric Kruskal-Wallis ANOVA.

C. Boxplot of mandibular PC1 data by mouse genotype. Numbers of mice per genotype are indicated. Statistical analysis was performed using a Tukey's HSD test.

D. Wireframes depicting mouse mandible side view and variations as a function of genotypes. Grey dots represent the consensus cranium shape, and black dots represent the shape depicted by minimum (upper

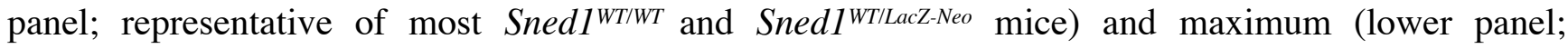

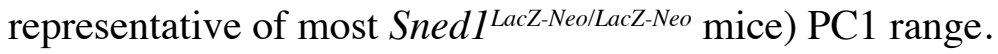

E. Pictures of a p1.5 wild-type (left panel) and age-matched dead Sned1 $1^{-/}$(right panel) neonates showing the absence of the lower jaw in the knockout pup.

\section{Figure 6. Patterns of expression of Snedl gene during embryogenesis}

A. Whole-mount $\beta$-galactosidase assay (LacZ staining) performed on heterozygous E11.5 Sned1 ${ }^{\text {WT/LacZ- }}$ ${ }^{N e o}$ embryo.

B. Sagittal section of the head region of LacZ-stained E11.5 LacZ Sned1 ${ }^{\text {WT/LacZ-Neo }}$ embryo. 1, 2, 3, and 4 represent the branchial arches; NP: nasal process; RP: Rathke's pouch.

C. Sagittal sections of LacZ-stained E11.5 LacZ Sned1 ${ }^{\text {WT/LacZ-Neo }}$ embryo (left panel: lateral section; right panel: across the midline). Lu B, lung bud; So, somites; DRG, dorsal root ganglia.

D. Whole-mount $\beta$-galactosidase assay (LacZ staining) performed on heterozygous E13.5 Sned1 ${ }^{\text {wT/LacZ- }}$ Neo embryo.

E. Transverse section of the head region of LacZ-stained E13.5 LacZ Sned1 ${ }^{\text {WT/LacZ-Neo }}$ embryo. DRG, dorsal root ganglia; Hu: humerus; MC: site of apposition in the midline of Meckel's cartilages; NS, cartilage primordium of nasal septum; Sc: blade of scapula; T, tongue; VO, vomeronasal organ.

F. Section of paw from LacZ-stained E13.5 LacZ Sned1 ${ }^{\text {WT/LacZ-Neo }}$ embryo. Arrows indicate interdigital spaces. 
G. Sagittal sections of Lac-Z-stained E13.5 LacZ Sned1 ${ }^{\text {WT/LacZ-Neo }}$ embryo (from left to right: lateral to the midline). CP, choroid plexus; DRG, dorsal root ganglia; FL, forelimb; HL, hindlimb; NS, cartilage primordium of nasal septum; T, tongue; V, cartilage primordium of the vertebrae.

\section{Figure 7. NCC-specific Snedl knockout results in craniofacial malformations}

A. PCR genotyping of genomic DNA isolated from tail samples of Sned ${ }^{\text {WT/NCC- mice. }}$

B. Dotplots represent the weight of one-month-old mice of the Sned1 ${ }^{\text {NCC- }}$ transgenic line. Numbers of mice per genotype are indicated. Lines represent the average weight of the genotype group. Statistical analysis was performed using an unpaired t-test.

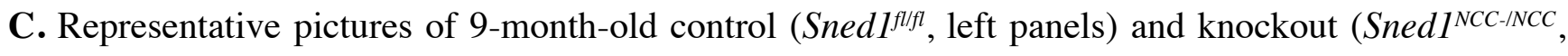
right panels) mice.

D. $\mathrm{MCT}$ scans of 9-month-old mice show examples of variation in skull morphology based upon genotype.

E-I. Examination of the skull of a cohort of 30 adult (7 to 15 -month-old) mice of the Sned ${ }^{\text {NCC- }}$ transgenic line.

E. Nasal suture length. Numbers of mice per genotype are indicated. Lines represent average length of the genotype group. Statistical analysis was performed using an unpaired t-test.

F. Scatterplot of PC2 and PC3 for variation in the mouse cranial morphology of the Sned ${ }^{\text {NCC- }}$ transgenic line. Statistical analysis was performed using a one-way non-parametric Kruskal-Wallis ANOVA.

G. Boxplot of cranial PC2 data by mouse genotype. Numbers of mice per genotype are indicated. Statistical analysis was performed using a Tukey’s HSD test.

H. Wireframes depicting mouse cranium side (left) and top (right) views and variation of the shape as a function of genotypes. Grey dots represent the consensus cranium shape, black dots represent the shape

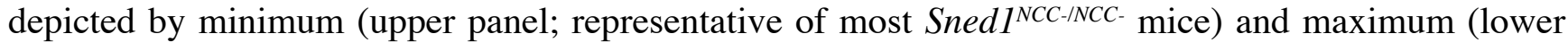
panel; representative of most Sned $1^{\text {WTffl }}$, Sned $1^{f l f l}$ and Sned $1^{W T / N C C-}$ mice) PC2 range. C: caudal; R: rostral. I. Occlusion and asymmetry assessment of the nasal cavity. Arrowhead indicates asymmetry. See also Supplementary Files 7-10.

\section{Figure 8. NCC-specific Sned1 knockout results in under-developed mandible}

A-C. Examination of the mandible of a cohort of 30 adult (7 to 15 -month old) mice of the Sned1 $1^{\text {NCC- }}$ transgenic line. 
A. Scatterplot of PC1 and PC3 for variation in the mouse mandibular morphology. Statistical analysis was performed using a one-way non-parametric Kruskal-Wallis ANOVA.

B. Boxplot of mandibular PC1 data by mouse genotype. Numbers of mice per genotype are indicated. Statistical analysis was performed using a Tukey's HSD test.

C. Wireframes depicting mouse mandible side view and variations as a function of genotypes. Grey dots represent the consensus cranium shape, and black dots represent the shape depicted by minimum (upper

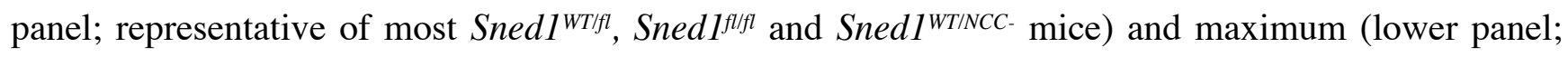

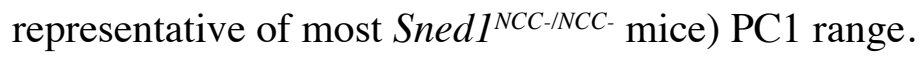

\section{Figure 9. Map of 2 q37.3 deletions in two patients with facial dysmorphic features}

A. Schematic representation of the human 2q37 chromosome region (dark blue box) showing two patients carrying a terminal deletion that includes the SNED1 locus (highlighted in light blue) but not $H D A C 4$. Deleted regions are indicated by horizontal black bars and gray bar indicates duplicated region in patient 14 from the Leroy at al, 2013 study. Source: UCSC genome browser: http://genome.ucsc.edu, GRCh38/hg38 Assembly.

B. Clinical features of two patients with 2q37.3 deletion including SNED1 but not HDAC4. 


\section{Supplementary Files}

Supplementary Method 1. Diagram representing the positioning of calvarial landmarks for geometric morphometric analysis

Supplementary Method 2. Diagram representing the positioning of mandibular landmarks for geometric morphometric analysis

\section{$\mu \mathrm{CT}$ scans used for occlusion and asymmetry assessment of nasal cavities.}

Supplementary File 1. File 1_WT_Adult.mp4: $\mu \mathrm{CT}$ scan of a 6-month-old wild-type (Sned ${ }^{W T / W T}$ ) mouse from the global Snedl knockout transgenic line.

Supplementary File 2. File 2_Het_Adult.mp4: $\mu$ CT scan of a 6-month-old heterozygous (Sned $1^{\text {WT/LacZ- }}$ $\left.{ }^{N e o}\right)$ mouse from the global Sned 1 knockout transgenic line.

Supplementary File 3. File 3_KO_Adult.mp4: $\mu$ CT scan of a 6-month-old knockout (Sned1 LacZ-Neo/LacZ${ }^{N e o}$ ) mouse from the global Sned1 knockout transgenic line.

Supplementary File 4. File 4_WT_Neonate.mp4: $\mu$ CT scan of a p0.5 wild-type (Sned1 ${ }^{W T / W T}$ ) pup from the global Sned 1 knockout transgenic line.

Supplementary File 5. File 5_Het_Neonate.mp4: $\mu \mathrm{CT}$ scan of a p0.5 heterozygous (Sned $1^{\text {WT/LacZ-Neo }}$ ) pup from the global Sned 1 knockout transgenic line.

Supplementary File 6. File 6_KO_Neonate.mp4: $\mu \mathrm{CT}$ scan of a p0.5 knockout (Sned1 ${ }^{\text {WT/LacZ-Neo }}$ ) pup from the global Snedl knockout transgenic line.

Supplementary File 7. File 7_NCC_WTfl_Adult.mp4: $\mu$ CT scan of a 9-month-old control (Sned $1^{\text {WTffl })}$ mouse from the NCC-specific Sned 1 knockout transgenic line.

Supplementary File 8. File 8_NCC_flfl_Adult.mp4: $\mu$ CT scan of an 8-month-old control (Sned $1^{f l f l}$ ) mouse from the NCC-specific Sned1 knockout transgenic line.

Supplementary File 9. File 9_NCC_Het_Adult.mp4: $\mu$ CT scan of a 9-month-old heterozygous $\left(\right.$ Sned $\left.1^{W T / N C C-}\right)$ mouse from the NCC-specific Snedl knockout transgenic line.

Supplementary File 10. File 10_NCC_KO_Adult.mp4: $\mu \mathrm{CT}$ scan of an 8-month-old knockout $\left(\right.$ Sned $\left.1^{N C C-/ N C C-}\right)$ mouse from the NCC-specific Snedl knockout transgenic line. 
Figure 2. Sned1 knockout strategies and genotyping

A.

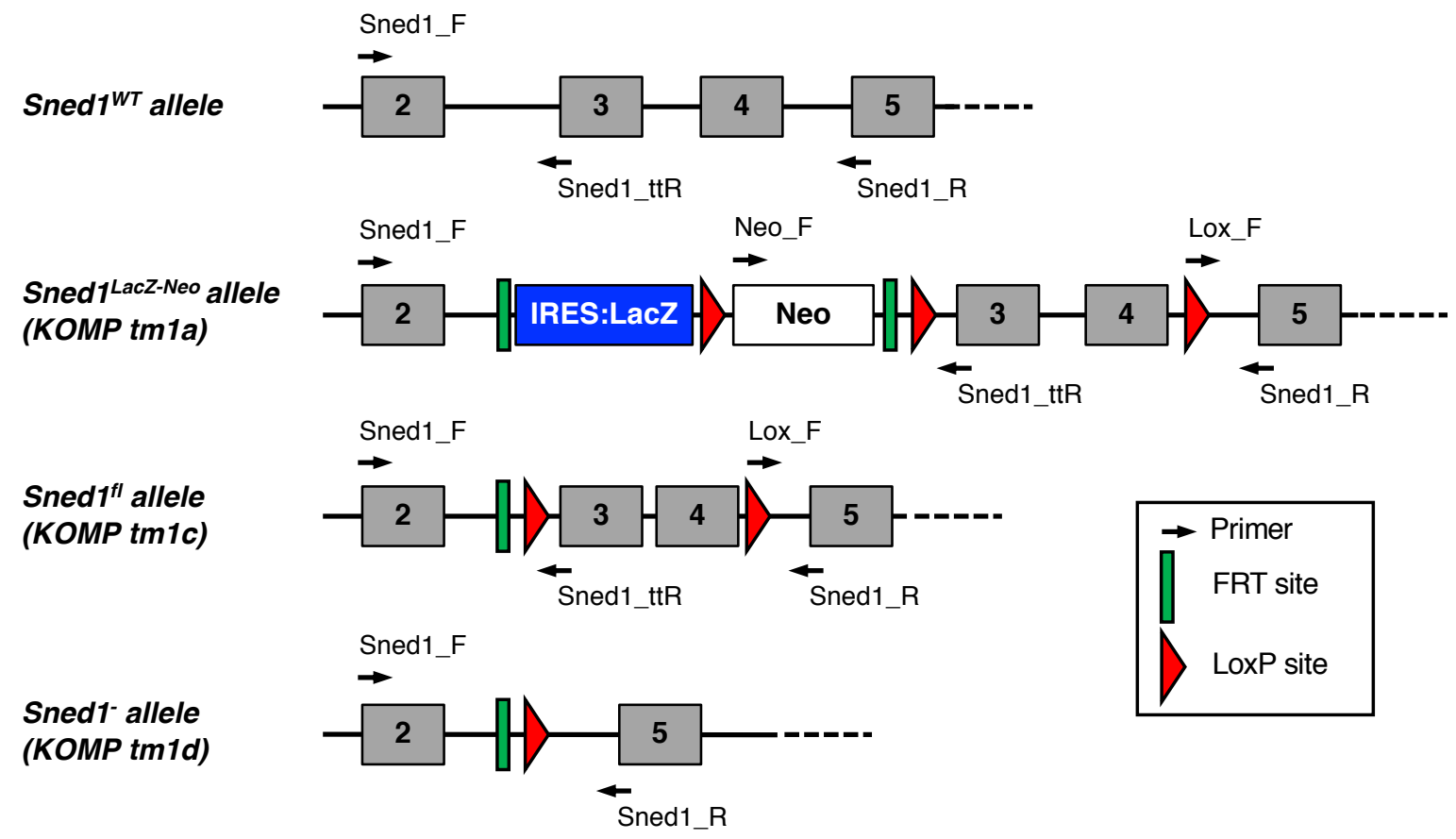

B.

Sned1 ${ }^{\text {WT/LacZ-Neo }}$

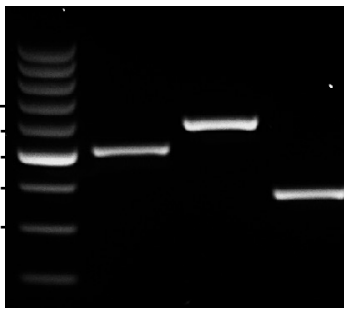

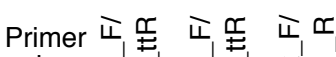

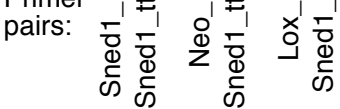

C.

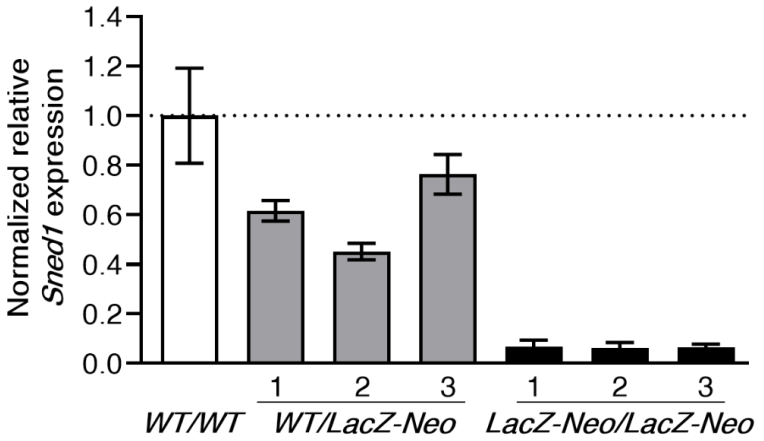

Sned1 ${ }^{W T / f I}$

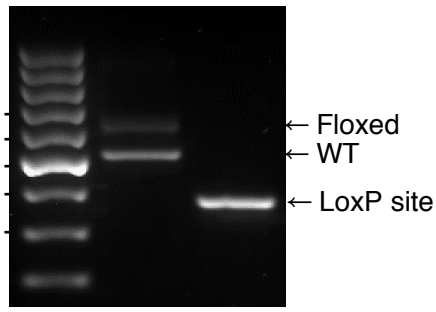

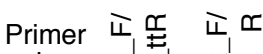

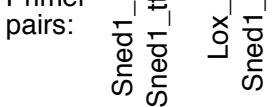

Sned1 ${ }^{W T /-}$

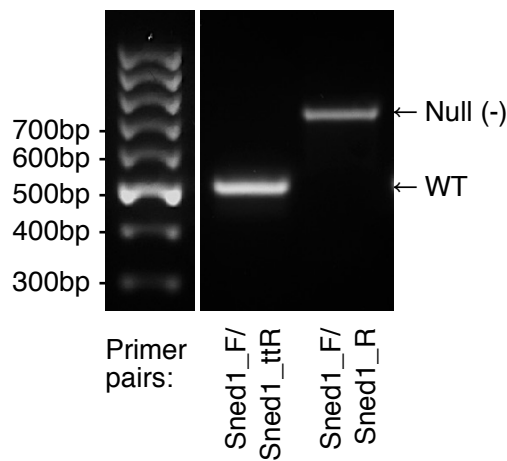

D.

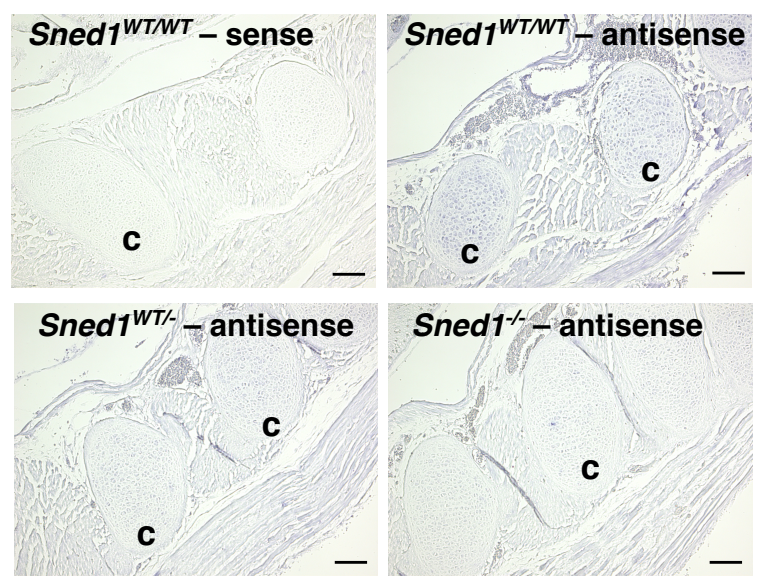


Figure 3. Surviving Sned1 knockout mice present growth defects

A.

B.
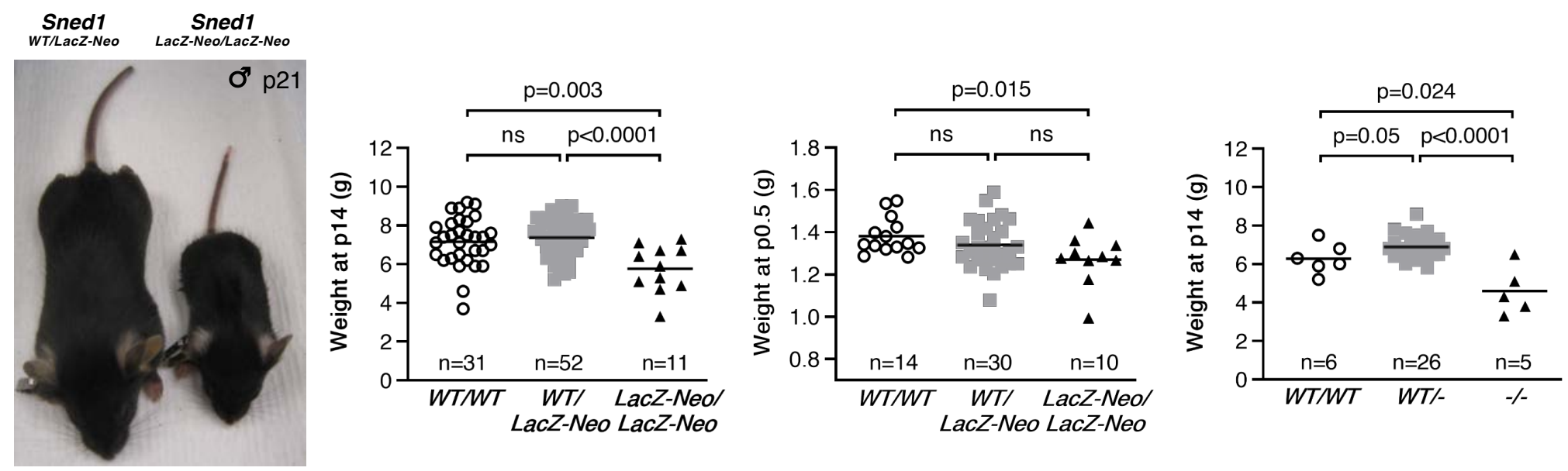

E.

Humerus

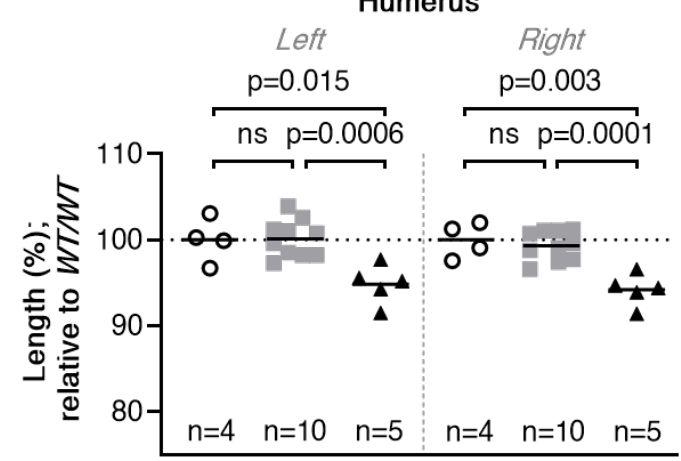

F.

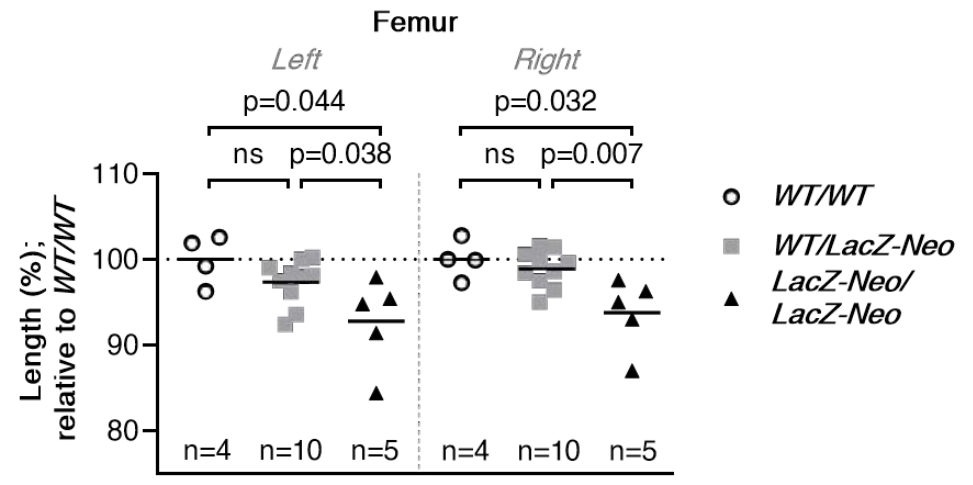


Figure 4. Sned1 knockout results in craniofacial malformations

A.

Sned1 ${ }^{W T W T}$ Sned1 ${ }^{\text {WT/LacZ-Neo }}$

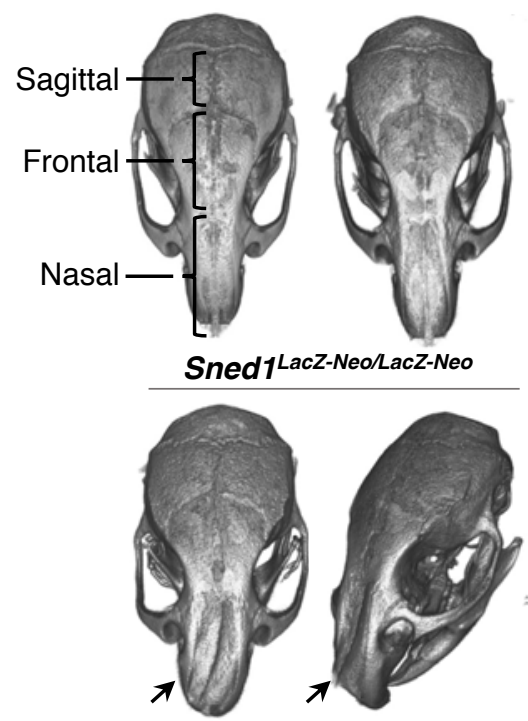

C.

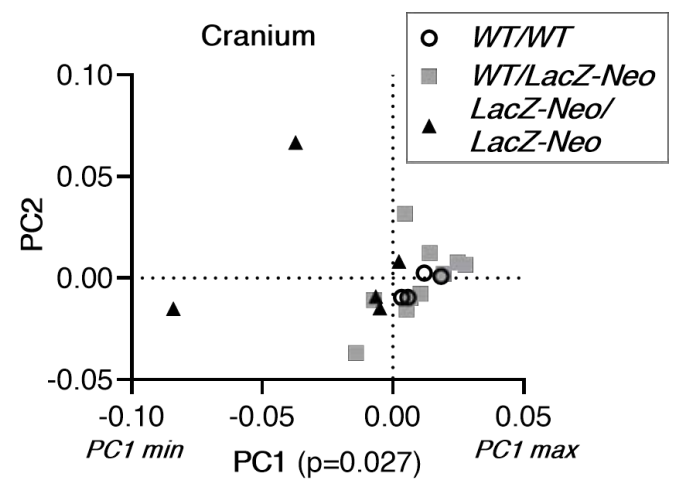

F.

WT/WT

졸

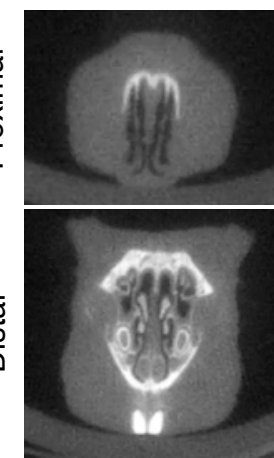

WT/LacZ-Neo LacZ-Neo/LacZ-Neo
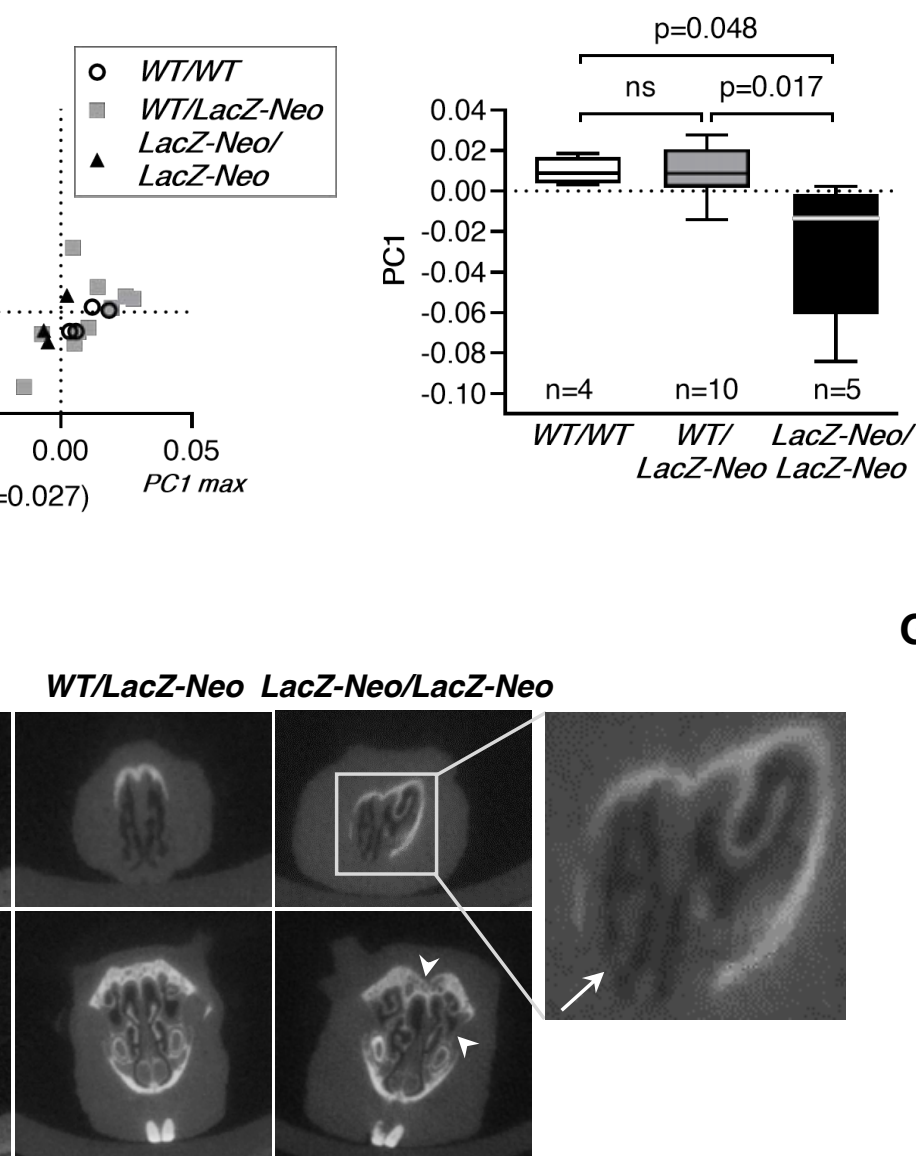

Frontal suture

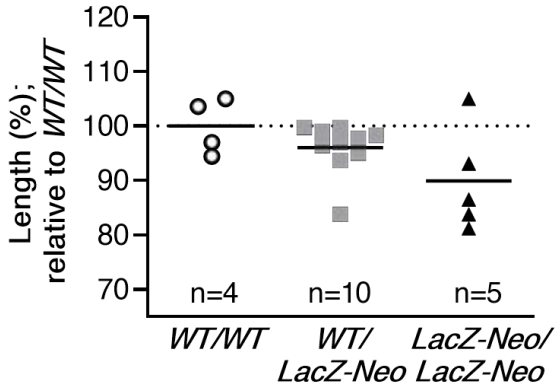

E.

PC1 Min
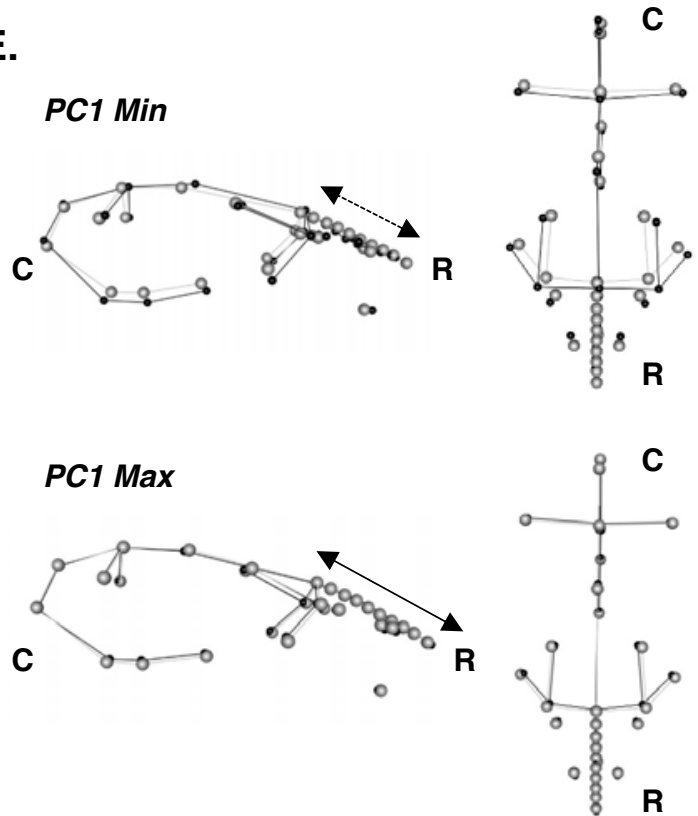

G.

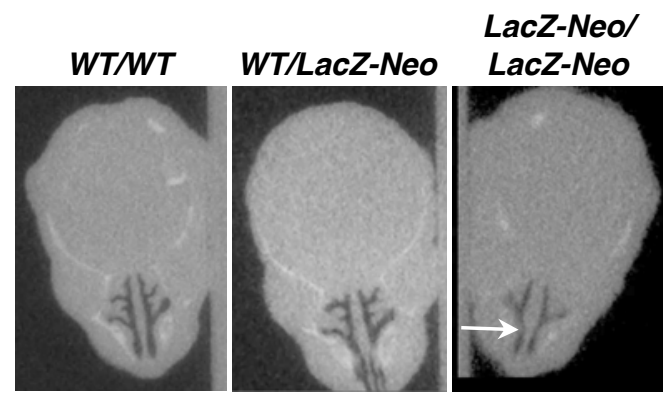

\begin{tabular}{|l|c|c|}
\hline Genotype (adult) & Occlusion & Asymmetry \\
\hline Sned1 ${ }^{\text {WT/WT }}$ & $0 / 4$ & $0 / 4$ \\
\hline Sned1 ${ }^{\text {WT/LacZ-Neo }}$ & $0 / 10$ & $1 / 10$ \\
\hline Sned1 ${ }^{\text {LacZ-Neo/LacZ-Neo }}$ & $\mathbf{2 / 5}$ & $\mathbf{3} / \mathbf{5}$ \\
\hline
\end{tabular}

\begin{tabular}{|l|c|c|}
\hline Genotype (p0.5) & Occlusion & Asymmetry \\
\hline Sned1 ${ }^{W T W T}$ & $0 / 4$ & $0 / 4$ \\
\hline Sned1WT/LacZ-Neo & $3 / 15$ & $2 / 15$ \\
\hline Sned1 LacZ-Neo/LacZ-Neo & $\mathbf{3 / 3}$ & $\mathbf{0} / 3$ \\
\hline
\end{tabular}


Figure 5. Sned1 knockout results in under-developed mandible

A.

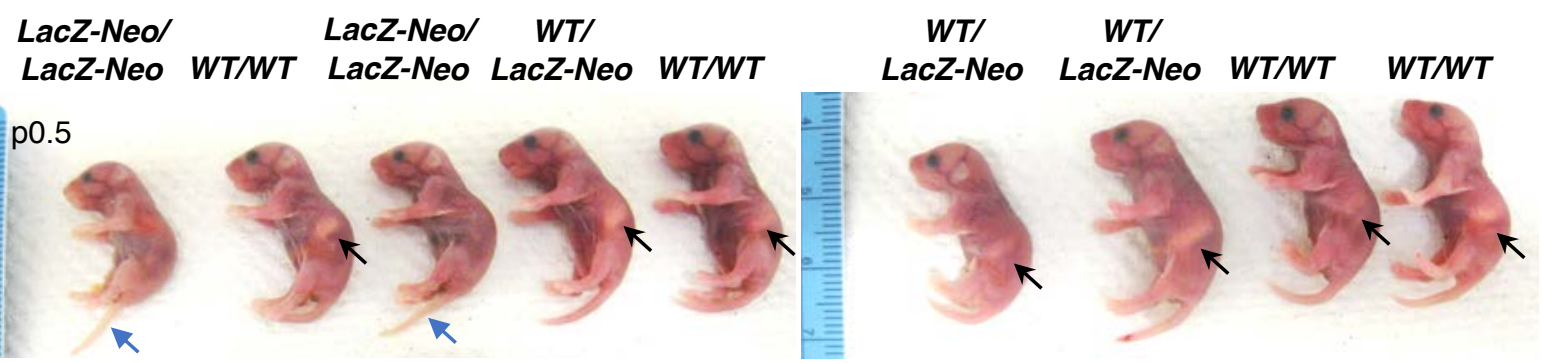

B.

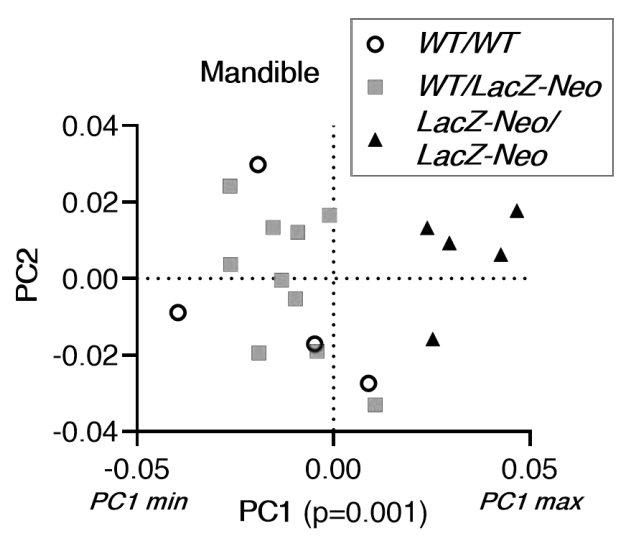

E.

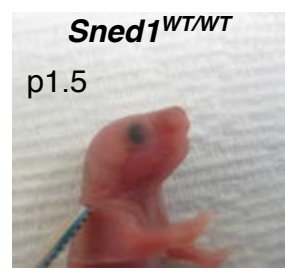

Sned1\%-
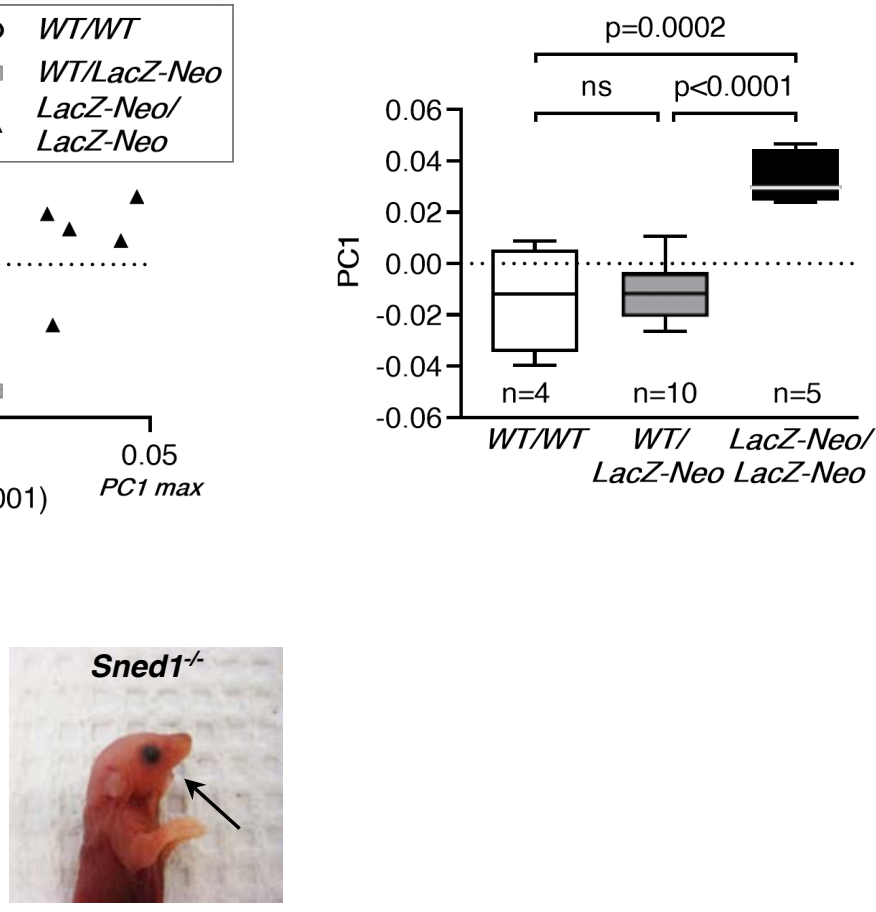

D.

PC1 Min

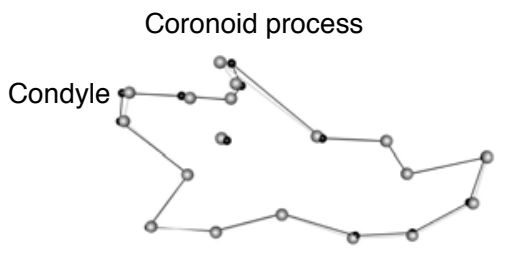

PC1 Max

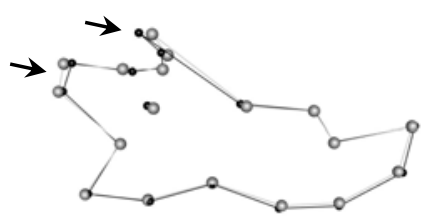


Figure 6. Patterns of expression of Sned1 gene during embryogenesis

A.

\section{Whole-mount}

$\beta$-galactosidase activity assay

Sned1 ${ }^{\text {WT/LacZ-Neo }}$ - E11.5

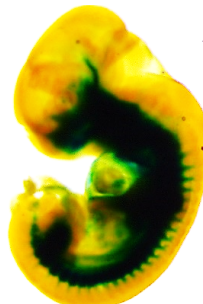

D.

Whole-mount

$\beta$-galactosidase activity assay Sned1 ${ }^{\text {WT/LacZ-Neo }-~ E 13.5 ~}$

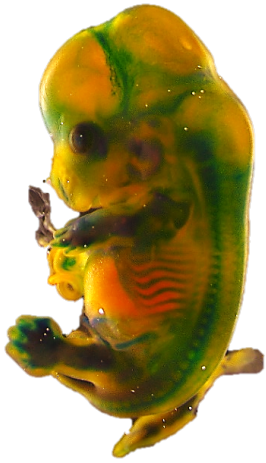

G.

E.

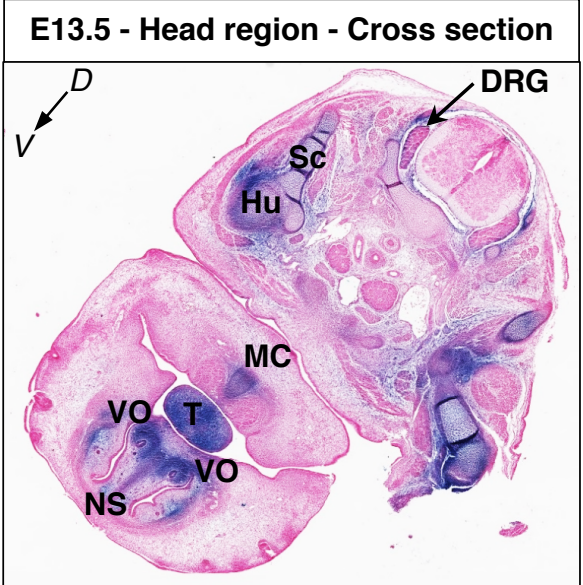

E11.5 - Sagittal section Lateral to Midline

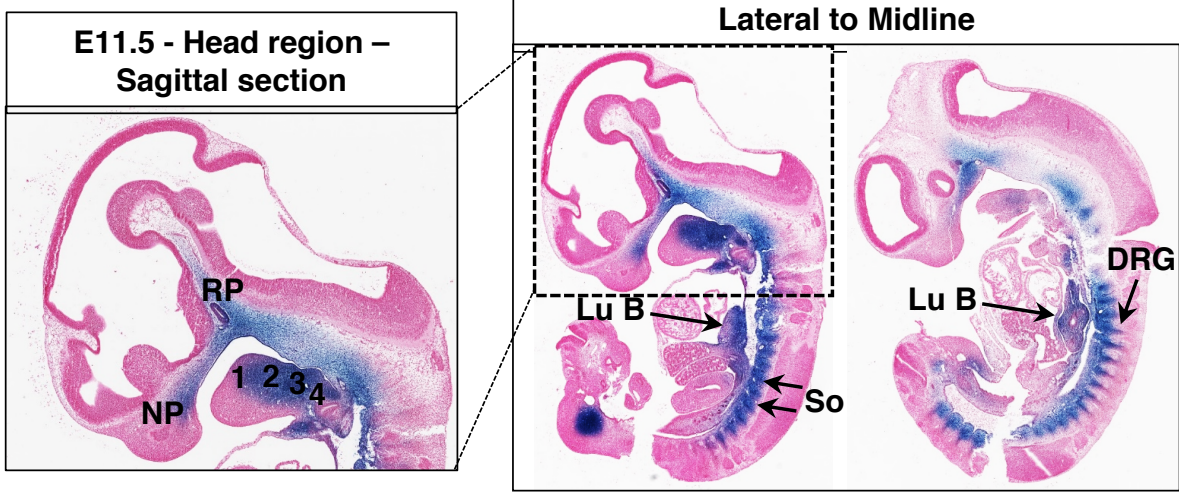

F.

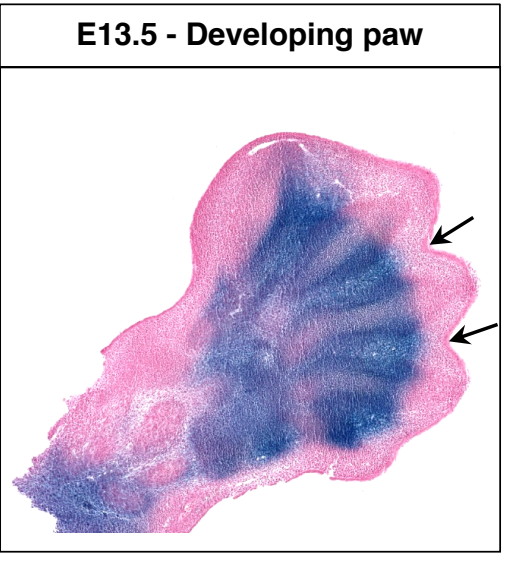

E13.5 - Sagittal section - Lateral to Midline
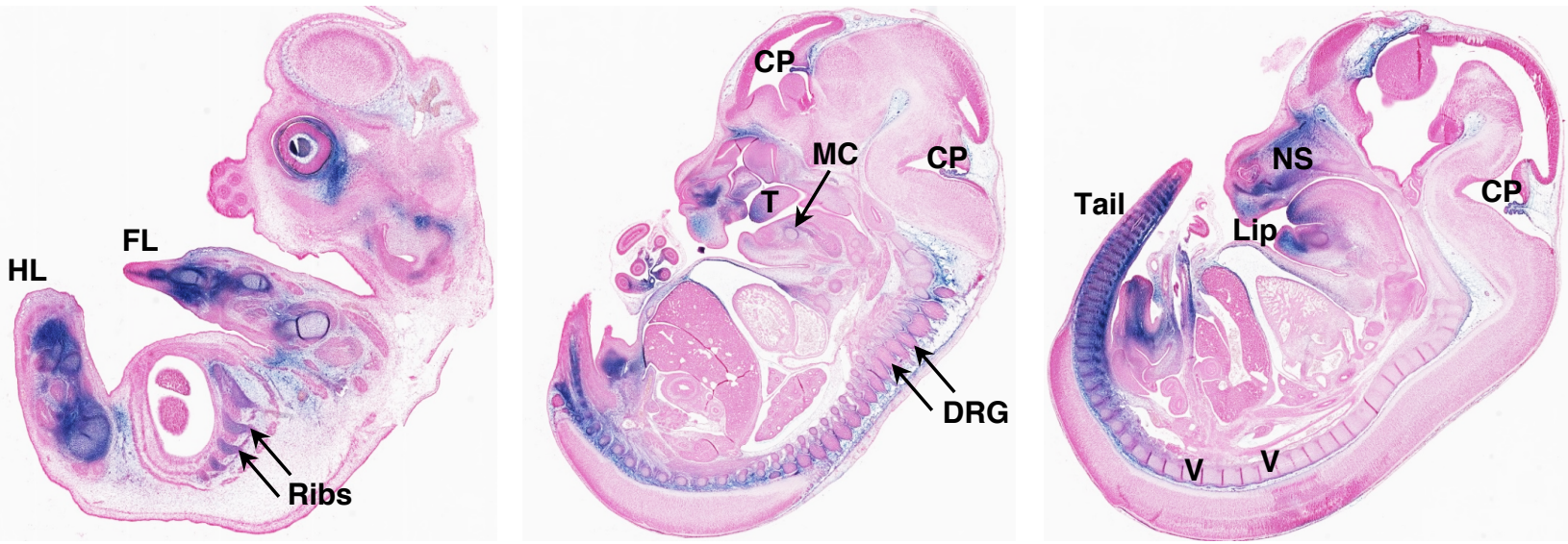
Figure 8. NCC-specific Sned1 knockout results in under-developed mandible

A.

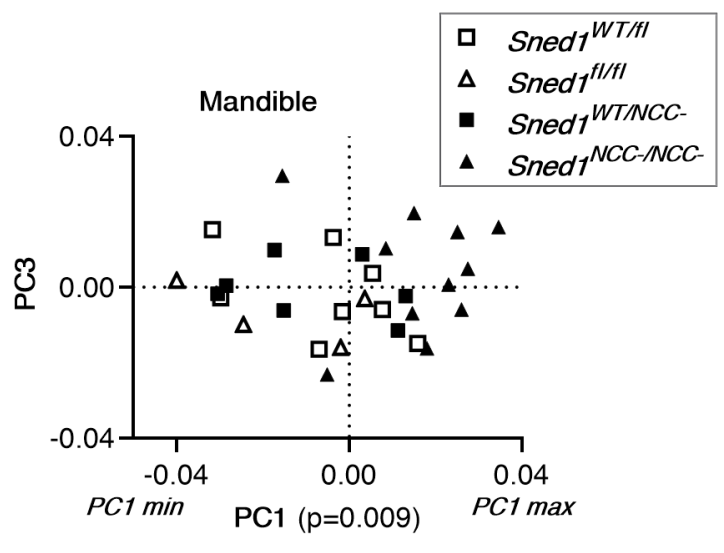

B.

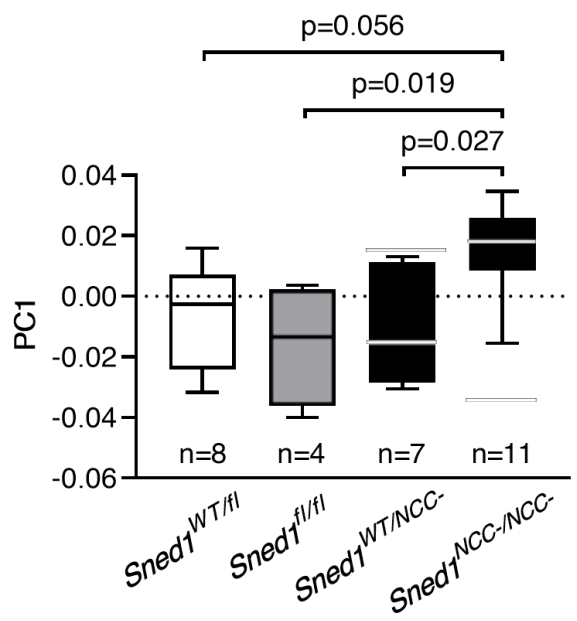

C.

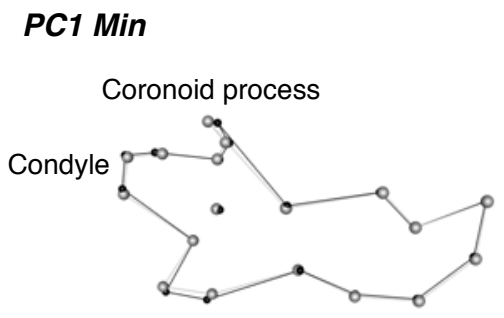

PC1 Max

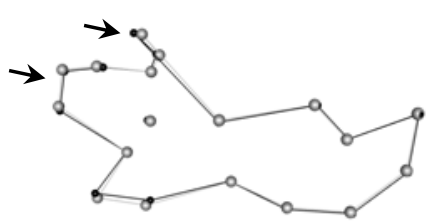


Figure 9. Map of $2 q 37.3$ deletions in two patients with facial dysmorphic features

A.

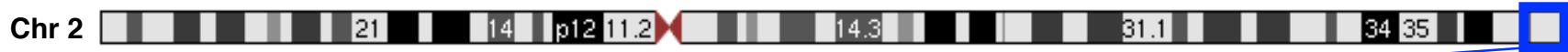

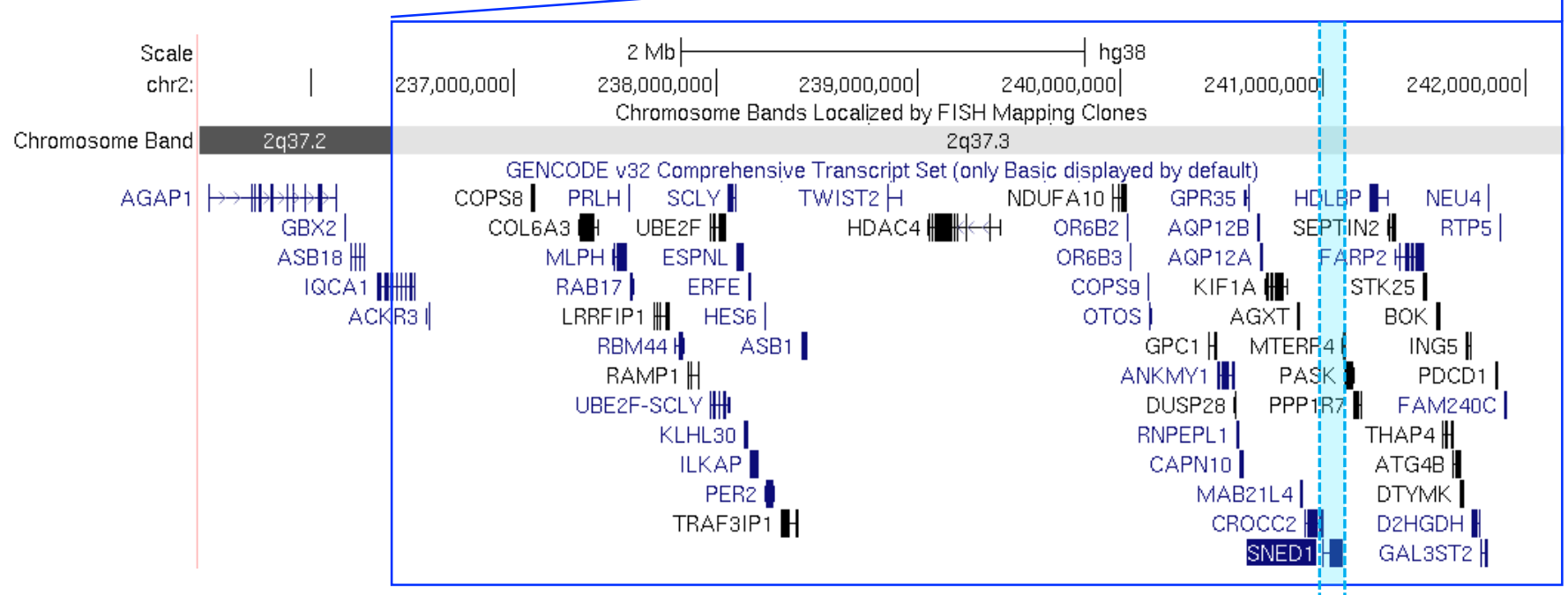

Williams et al. (2010)

Patient 2282

Leroy et al. (2013)

Patient 14

B.

\begin{tabular}{|l|cc|}
\hline \multicolumn{1}{|c|}{ Patient } & (Williams et al. 2010) & \multicolumn{1}{c|}{$\begin{array}{c}\text { 2282 } \\
\text { (Leroy et al. 2013) }\end{array}$} \\
\hline Cytogenetic findings & del(2)(q37.3) & der(2)dup(2)(q37.2q37.3); del(2)(q37.3) \\
\hline Sex & $\mathrm{F}$ & $\mathrm{M}$ \\
\hline Age & 7 years & 7 years \\
\hline Developmental delay & + & + \\
\hline Motor delay & + & - \\
\hline Overweight & + & - \\
\hline Facial features & Broad face, midface hypoplasia & $\begin{array}{c}\text { Frontal bossing (prominent forehead), } \\
\text { flattened philtrum, the groove between } \\
\text { the nose and the upper lip }\end{array}$ \\
\hline Other & Autistic behavior, seizures & $\begin{array}{c}\text { Broader big toe, joint hypermobility, long } \\
\text { fingers }\end{array}$ \\
\hline
\end{tabular}

